Revue des patrimoines

16 | 2011

Le patrimoine militaire et la question urbaine

\title{
Le patrimoine militaire de la rade de Toulon : histoire, territorialité et gestion patrimoniale
}

\section{Bernard Cros}

\section{(2) OpenEdition}

\section{Journals}

Édition électronique

URL : http://journals.openedition.org/insitu/275

DOI : 10.4000/insitu. 275

ISSN : 1630-7305

Éditeur

Ministère de la Culture

Référence électronique

Bernard Cros, «Le patrimoine militaire de la rade de Toulon : histoire, territorialité et gestion patrimoniale », In Situ [En ligne], 16 | 2011, mis en ligne le 27 juin 2011, consulté le 21 décembre 2020. URL : http://journals.openedition.org/insitu/275 ; DOI : https://doi.org/10.4000/insitu.275

Ce document a été généré automatiquement le 21 décembre 2020.

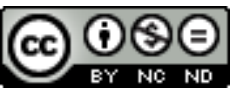

In Situ Revues des patrimoines est mis à disposition selon les termes de la licence Creative Commons Attribution - Pas d'Utilisation Commerciale - Pas de Modification 4.0 International. 


\title{
Le patrimoine militaire de la rade de Toulon : histoire, territorialité et gestion patrimoniale
}

\author{
Bernard Cros
}

\section{Une rade faite pour la défense}

Entre le Rhône et le Var, le littoral provençal ne constitue un «bastion » du royaume de France qu'après le rattachement de la Provence à la France en 1481. Le roi de France est désormais Comte de Provence; il prend la pleine mesure de la valeur stratégique de ce territoire lors des guerres d'Italie. La côte provençale est généralement découpée et marquée par un relief qui rend son accès maritime peu praticable (fig. $\left.\mathbf{n}^{\circ} \mathbf{1}\right)$. Quelques longues baies ou rades profondes l'échancrent de place en place: rade d'Antibes, Golfe Juan, golfe de Grimaud, rade d'Hyères. Elles sont néanmoins ouvertes aux houles du large et sujettes aux vents qui les balaient. La rade de Villefranche est d'assez bonne tenue, mais elle relève du duc de Savoie puis de l'Italie jusqu'en 1860. La rade de Toulon fait figure d'exception dans ce panorama. Particulièrement vaste (plus de 1500 ha), elle est bordée de rivages sur les trois quarts de son périmètre et bénéficie d'une protection naturelle contre les houles du large. Celles de sud-est, dominantes, sont atténuées par les îles d'Hyères et le tombolo de Giens. Le vent dominant du nord-est (le mistral) y voit ses effets atténués par le faible essor qu'il y prend. De telles qualités ne pouvaient que favoriser l'essor de la navigation à partir ou à destination de Toulon. Son port commerce ainsi avec la péninsule scandinave, les îles britanniques et Terre-Neuve, tout autant qu'avec le bassin méditerranéen. 


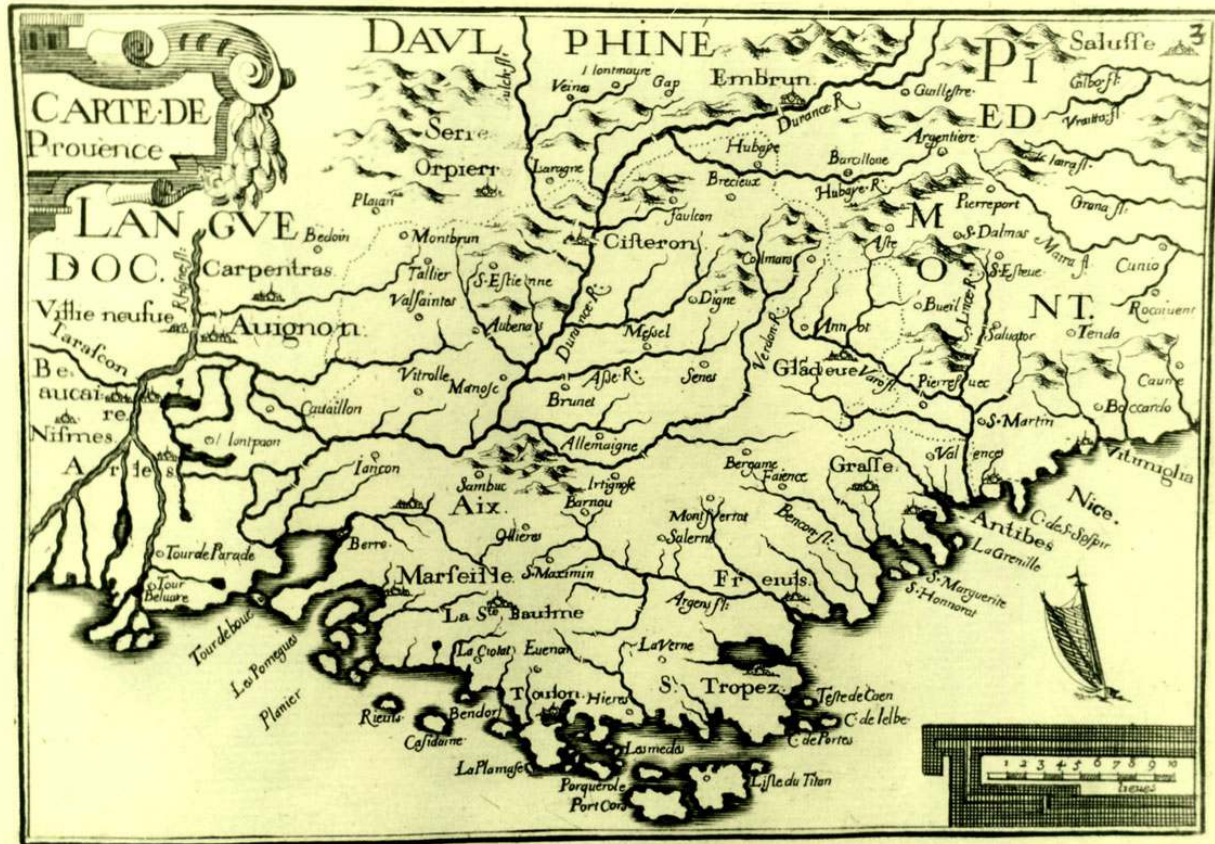

Carte de Provence publiée par Tassin dans Plans et profilz des principales villes de la province de Provence, vers 1634 (Coll. Auteur) Phot. B. Cros.

2 Lorsque les Toulonnais réalisent des travaux afin de gagner sur la mer au niveau du port, à la fin du XVI ${ }^{e}$ siècle, le roi Henri IV leur concède la libre jouissance des terrains ainsi acquis. Les lettres patentes qu'il délivre à cet effet en 1595 sont enregistrées en 1599 par le Parlement de Provence, qui stipule qu'une place sera réservée pour la «fabrique des vaisseaux ». On peut y voir l'acte fondateur d'un port de guerre à Toulon. Les galères seront d'ailleurs basées à Toulon en 1610. Mais c'est avec Richelieu que la notion de marine d'État, royale et permanente, prend tout sens. Mettant de l'ordre dans une institution encore balbutiante et considérée comme source de prébendes, le cardinal ministre initie pour la première fois une véritable politique maritime pour la France.

\section{Du Parc de marine à l'arsenal}

Le « Parc de marine », berceau du futur grand arsenal, est implanté en partie nord-ouest de la darse créée par l'ingénieur de Bonnefons au début du XVII ${ }^{e}$ siècle $^{1}$ (fig. $\mathbf{n}^{\circ} \mathbf{2}$ ). Un chantier de construction navale occupe le terre-plein maritime. Des magasins et ateliers sont édifiés le long du rempart qui couvre la darse face à la petite rade. Aménagé au fil du temps et au gré des besoins, sans plan d'ensemble, le parc s'étend progressivement vers la ville, utilisant le rare espace disponible entre le rempart et le dense tissu urbain de Toulon. C'est rapidement un ensemble disparate, qui se mélange à la ville et qui souffre d'une mauvaise fonctionnalité tout en étant exposé aux chapardages en tous genres. «Il n'y avait en ce temps-là aucun arsenal, mais seulement quelques petits magasins fermés, avec des ateliers qui ne l'étaient point du tout, en sorte que les particuliers y allaient prendre furtivement ce qui leur convenait ... Mr Colbert, pour remédier à cet inconvénient, fit rassembler dans un même lieu tous ces différents magasins par une 
enceinte de murailles, qui fut appelé parc. Il n'y avait qu'une seule porte, gardée par des Suisses ", écrira plus tard l'intendant de la marine Usson de Bonrepaus.

Figure 2

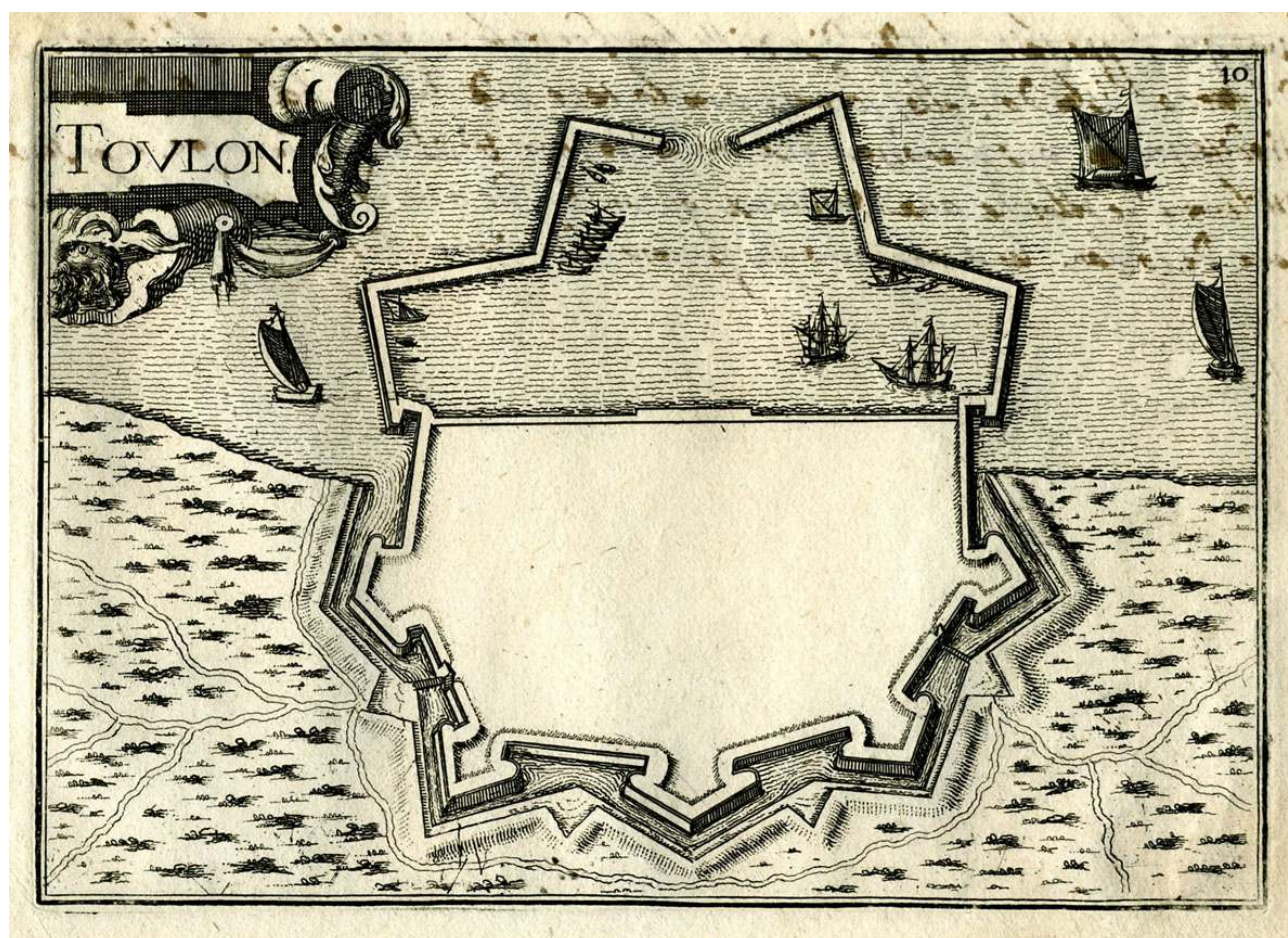

Toulon, par Tassin dans Plans et profilz des principales villes de la province de Provence, vers 1634 (Coll. Auteur). Phot. B. Cros.

4 En 1670, l'intendant Matharel s'efforce de gagner en commodité, rationalité et sécurité. Il fait construire, hors les murs, un "petit parc ", sorte d'annexe dotée de halles pour les bois de mâture et de construction et de divers ateliers et magasins. Ce n'est qu'un pisaller, dans l'attente d'un projet d'agrandissement de l'arsenal digne de sa vocation. Colbert souhaite en effet tenir à Toulon une escadre de cinquante à soixante vaisseaux, pour marquer la place prépondérante que le royaume de France entend tenir en Méditerranée. Ce besoin d'extension est formulé par Colbert à une époque où Rochefort est le siège d'un des plus grands chantiers royaux dans le pays. Le concept d'arsenal étant encore du domaine du "vernaculaire", Colbert entend faire de Rochefort un champ d'études qui profitera aux autres ports de guerre. À mesure que l'arsenal de Rochefort s'édifie, Colbert échange effectivement avec ses intendants afin d'élaborer les règlements qui fixeront le fonctionnement des arsenaux maritimes. À Toulon, il faudra pratiquement dix années pour aboutir à un projet d'arsenal qui satisfasse Colbert. " Nous ne sommes pas en un règne de petites choses ... il est impossible que vous puissiez imaginer rien de trop grand », déclare Colbert au chevalier de Clerville lorsqu'il est missionné pour arrêter le projet de grand arsenal toulonnais. Le rigoureux ministre précise toutefois que ceci « doit toutefois avoir sa proportion » ...

5 Les projets succèdent aux projets. Les ingénieurs ou architectes Clerville, Puget, Gombert, en fournissent pratiquement dix. Seignelay emmène avec lui un ingénieur qui s'avère ne pas connaître grand-chose à la marine! L'intendant Arnoul se prend lui-même pour un ingénieur et y va de ses projets. Rien n'y fait. Les projets semblent par trop confus à 
Colbert ; surtout, ils sont d'un coût qui lui parait - comme souvent - exorbitant. Il faut qu'Arnoul en vienne à embrouiller la matière pour que Colbert décide d'appeler Vauban à la rescousse, en 1679. De fait, Vauban est désormais dans son rôle, puisqu'il a succédé à Clerville depuis un an comme Commissaire général des fortifications. Il s'est illico illustré en travaux maritimes à Dunkerque, qu'il a dotée du chenal qui lui faisait défaut. Non seulement par manque d'envergure des ingénieurs, mais aussi et surtout en raison du sens trop poussé de Colbert pour les économies. En trois semaines, Vauban démêle l'écheveau toulonnais. Son puissant sens de la synthèse, joint à une grande force de persuasion, lui permet d'enlever la décision. Toulon entre alors dans une vague de grands travaux qui dureront quinze ans pour l'essentiel. Une nouvelle darse est creusée à l'ouest de la darse de Henri IV ; exclusivement affectée à la marine royale, elle est bordée des chantiers et édifices propres à la construction et au soutien d'une puissante flotte de guerre. L'arsenal est doté des attributs propres à un grand port de guerre : une corderie longue de $400 \mathrm{~m}$ et accompagnée de ses magasins aux cordages et au goudron, ainsi qu'une étuve. Un élégant magasin général, un parc d'artillerie avec grande halle aux affûts et parcs aux canons et boulets participent au soutien logistique de la flotte. Halles aux mâts, forges et ateliers de toutes sortes jouxtent le chantier de construction navale, situé au cœur géographique et fonctionnel de l'arsenal. Les môles bastionnés qui ceinturent la nouvelle darse se couvrent de magasins pour les vaisseaux. À l'occasion de ces grands travaux d'aménagement, la ville s'agrandit aussi vers l'ouest, à l'abri d'une enceinte bastionnée qui protège la ville et l'arsenal. Car c'est une constante toulonnaise ; la ville vit au rythme de « son » arsenal et de « sa » marine.

\section{Toulon place forte maritime}

6 Tant que dure la marine à voiles, l'arsenal se tient et se développe à l'intérieur du corset de pierre dont Vauban le dote. Toulon devient de fait un objectif stratégique pour les adversaires de la France en Méditerranée, au premier rang desquels les Anglais. Le littoral circonvoisin se couvre ainsi d'ouvrages de défense côtière destinés à interdire toute incursion navale dans la petite rade. La presqu'île de Saint-Mandrier n'échappe pas à cette « frénésie architecturale » : clef de la grande rade $^{2}$, elle en contrôle les approches et offre des plages de descente ou de débarquement dont les défenseurs de Toulon doivent impérativement conserver la maitrise (fig. $\mathbf{n}^{\circ} \mathbf{3}$ ). Des batteries et des forts côtiers se dressent à chaque pointe ou cap dont le rivage est abondamment garni. À la veille de l'attaque conduite en 1707 par le duc de Savoie, en pleine guerre de succession d'Espagne, 150 canons répartis dans 28 ouvrages côtiers veillent à la sécurité du port de guerre. Ce front de mer s'étend du cap Carqueiranne à Bandol, en passant par Saint-Mandrier et l'isthme des Sablettes. La frontière étant alors sur le Var, la défense terrestre de la place n'est pas à négliger. Manifestement insuffisante durant les événements de 1707, la protection de Toulon connaît un vaste programme d'améliorations au cours du XVIII ${ }^{e}$ siècle. Une ligne de forts et redoutes est tracée à l'est de la ville, entre la hauteur de Lamalgue et le bas du Mont Faron. À l'ouest, quelques redoutes sont construites de part et d'autre de la vallée du Las, pour empêcher un contournement du Faron par le nord, en vue de prendre les défenseurs à revers. Ainsi, à la fin du XVIII ${ }^{\mathrm{e}}$ siècle, l'existence du port de guerre de Toulon se traduit par la présence d'ouvrages défensifs tant sur les hauteurs environnant la ville, que sur les proéminences du rivage. La vocation militaire de Toulon s'inscrit ainsi autant dans son urbanisme (enceinte urbaine bastionnée, arsenal juxtaposé 
à la ville) que dans son paysage côtier et rural. Au « visible » (ouvrages militaires) s'ajoute un "poids invisible», celui des servitudes imposées par les ouvrages militaires: servitudes non cedificandi autour des remparts et dans le champ de tir des pièces d'artillerie.

Figure 3

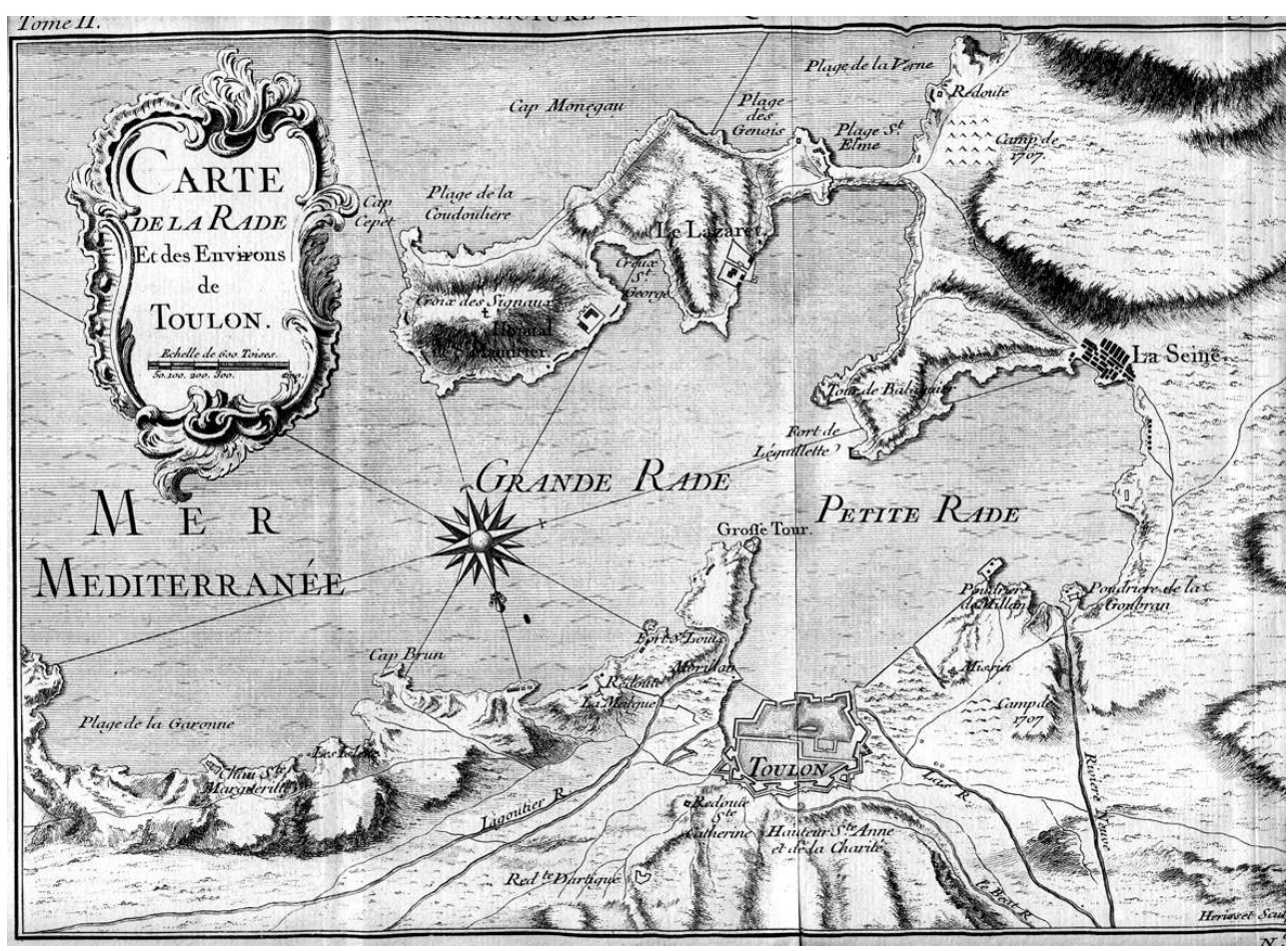

Carte de la rade et des environs de Toulon, publiée par Bélidor dans L'architecture hydraulique, seconde partie tome second, pl. IV, en 1753. (Coll. Auteur) Phot. B. Cros.

\section{L'expansion littorale}

$7 \mathrm{Au}$ cours du XVIII e siècle, l'arsenal est pourvu des moyens qui lui faisaient défaut au terme du chantier, inachevé, lancé par Vauban. La première forme de radoub française en Méditerranée est mise en service en 1778. La boulangerie neuve, construite en 1700, voit ses capacités décuplées avec la création d'une aile complémentaire au début des années 1780. À la fin du règne de la voile, l'arsenal est perçu comme étriqué face aux besoins grandissants de la marine. L'ère est aux expéditions lointaines, coloniales ou scientifiques. Les théories de construction navale militent pour une conservation des vaisseaux sur cale, inachevés et en attente de mise à flot au moment voulu. Un nouvel agrandissement de l'arsenal vient à l'ordre du jour en 1821. Comme au temps de Colbert, les réflexions vont durer de nombreuses années. Faut-il agrandir vers l'ouest, ce qui est logique mais se heurte aux contraintes techniques induites par la médiocrité du sous-sol? Ou bien vers l'est, ce qui est antinomique avec la présence de la ville, mais techniquement plus simple? Un pis-aller (une fois de plus!) est exploité, avec la construction, au Mourillon, d'un établissement autonome de construction navale.

8 À la même époque, la marine étend son implantation en presqu'île de Saint-Mandrier. La vieille infirmerie royale Saint-Louis, construite en 1669 pour soigner les marins et soldats 
de retour de campagne, est alors délabrée. Après avoir envisagé de la réhabiliter, la marine retient le parti d'une reconstruction complète. L'hôpital pavillonnaire qui la remplace est construit entre 1819 et 1830 par les forçats du bagne de Toulon; il est un élément marquant du littoral à l'entrée de la rade toulonnaise ${ }^{3}$ (fig. $\left.\mathbf{n}^{\circ} \mathbf{4}\right)\left(\mathbf{f i g} \cdot \mathbf{n}^{\circ} 5\right)$.

Figure 4

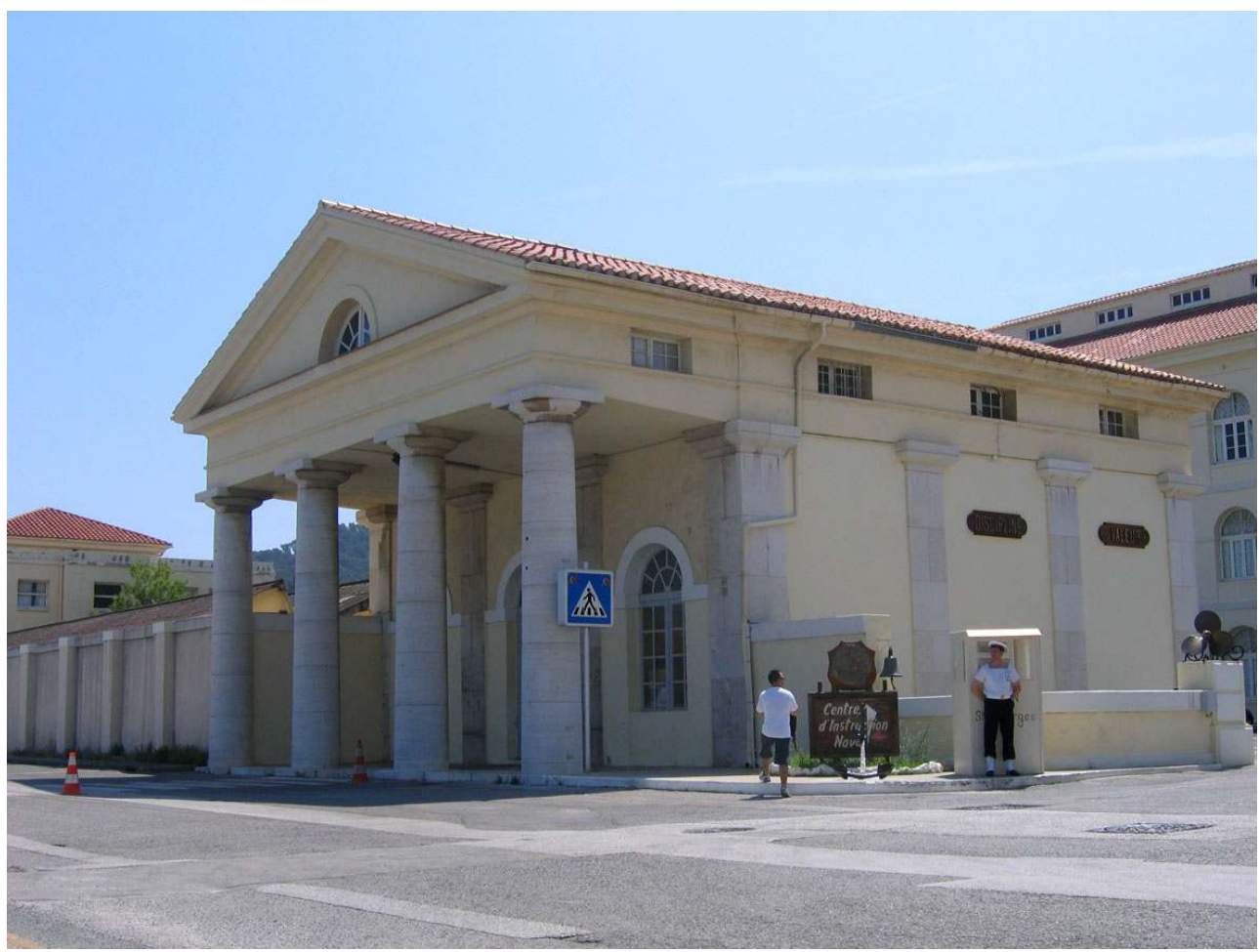

Corps de garde de l'ancien hôpital de Saint-Mandrier. Phot. B. Cros. 
Figure 5

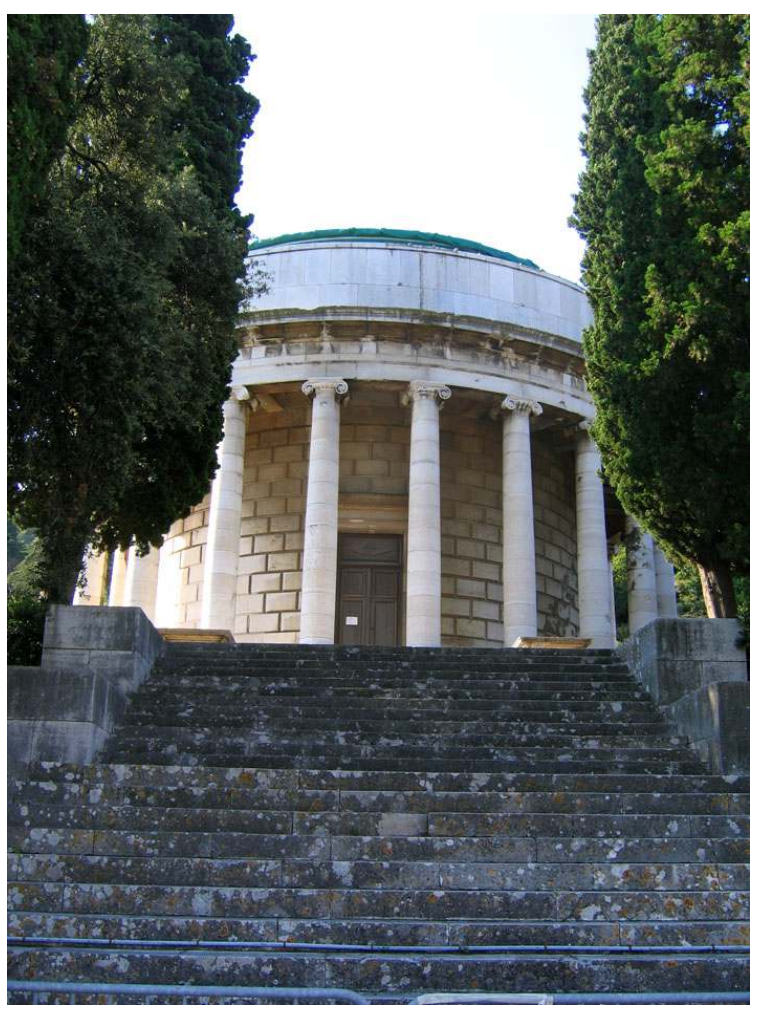

Chapelle de l'ancien hôpital de Saint-Mandrier. Phot. B. Cros.

9 La véritable modernisation de l'arsenal prend corps dès les débuts de la révolution industrielle. Celle-ci bouleverse la marine de guerre, dont les vaisseaux seront désormais construits en fer, propulsés à la vapeur et dotés d'une puissance de feu inédite. Le temps n'est plus au remaniement des installations portuaires à périmètre constant. C'est vers l'ouest que l'arsenal doit s'agrandir. L'établissement maritime est contraint de se mécaniser pour construire et entretenir la marine moderne. Aux ouvriers « en bois » vont bientôt se superposer, puis se substituer les ouvriers "en fer ». Par ordonnance du 12 septembre 1841, Louis-Philippe entérine les propositions des ministres de la Marine et de la Guerre, en vue d'agrandir l'arsenal dans la plaine de Castigneau, et déclare d'utilité publique l'acquisition des terrains concernés.

10 Le projet est essentiellement centré sur une nouvelle darse de $250 \mathrm{~m}$ de long à l'ouest de la darse Vauban et en abord de laquelle sont disposés de vastes terre-pleins industriels et portuaires. La construction navale doit ainsi bénéficier d'un ensemble d'ateliers pour les bâtiments à vapeur: fonderie, ajustage, montage, forges et chaudronnerie. Pour la réparation, on prévoit un bassin de radoub et une cale de halage. Pour l'équipement et le ravitaillement de la flotte sont prévus : un parc aux charbons, un parc aux subsistances et des casernes pour les équipages, l'infanterie de marine et l'artillerie de marine). La maîtrise foncière préalable nécessitera ainsi d'exproprier 85 parcelles, impliquant 23 propriétaires.

11 Par une nouvelle ordonnance du 23 décembre 1843, Louis-Philippe amende le tracé et l'emprise de l'arsenal de Castigneau, qui doit offrir un agrandissement de 37 ha. La mise au point du projet s'avèrera laborieuse. Les exigences respectives de la marine et du génie sont en effet difficiles à concilier. Les fortificateurs sont animés par le souci permanent de 
conserver l'intégrité et l'efficacité de l'enceinte défensive. Les études du génie sont en outre conditionnées par l'éventuel agrandissement de la ville de Toulon vers le nord, demandé par le conseil général du Var et approuvé en principe par le ministère de la Guerre en décembre 1843. Le devis de l'arsenal de Castigneau monte à près de 13 millions, en y incluant les travaux de fortification. Après les remous de la révolution de 1848 , les études concrètes reprennent en 1851, en butant sur les conditions d'aménagement du front de mer. Il est prévu d'aménager la limite sud de l'établissement de Castigneau en parc aux charbons et parc aux ancres, de façon à faciliter les mouvements entre la terre et les bords de mer.

12 Après optimisation de l'utilisation de l'emprise, les travaux entrent dans une phase active. La nouvelle darse de Castigneau prend sa configuration actuelle : elle dessert trois grands bassins de radoub, dont un double, construits entre 1855 et 1869. Pour permettre l'embarquement rapide d'un corps d'armée de 40000 hommes avec 7700 chevaux et 900 voitures, le front de Castigneau est pourvu de 9 appontements permettant l'amarrage de 18 navires de transport. Le ravitaillement des bâtiments de la flotte est facilité par un immense parc aux charbons de 20000 tonneaux de capacité. Les vivres de la flotte sont traités au sein d'un vaste établissement des subsistances édifié en 1857. Les terre-pleins voient s'édifier tout un ensemble d'ateliers de type industriel : l'atelier des chaudières doté d'une bascule pouvant peser des pièces jusque 40 tonnes, la fonderie à huit fourneaux Wilkinson, d'une capacité totale de 40 tonnes, l'atelier d'ajustage. Les machines à vapeur qui équipent ces ateliers crachent leurs fumées par une unique cheminée de $70 \mathrm{~m}$, la plus haute d'Europe.

13 Après 1852, c'est toute l'agglomération toulonnaise qui entre dans une ère de grands remaniements. L'agrandissement de l'enceinte urbaine est finalement décidé par décret du 28 septembre 1852, sur les bases du projet rédigé par le colonel Picot en 1844. Au début de l'année 1855, le conseil d'amirauté entrevoit le besoin de porter les limites de l'arsenal au-delà de celles de Castigneau, car la généralisation de la marine à vapeur laisse prévoir l'insuffisance d'espaces. La guerre de Crimée se traduit en effet par de nombreuses opérations de transports de troupes et de ravitaillements. La quantité et la variété des navires à amarrer dans le port ne cesse de croître, qu'il s'agisse de bâtiments de guerre ou d'auxiliaires en tous genres. En outre, la transition entre marine en bois et marine en métal ne se fait que progressivement. L'insuffisance des casernes et leur dissémination en ville ne facilitent pas le transit des troupes. Pour ces raisons, la marine demande que l'arsenal s'agrandisse jusque dans la plaine de Missiessy, alors même que Castigneau n'est pas achevé. Ce nouvel accroissement devra être accompagné du creusement d'une nouvelle darse au pied du château de Missiessy. Un premier projet est établi en 1855, dont l'emprise de 55 ha est couverte par une enceinte bastionnée couronnant les hauteurs de Missiessy et se refermant sur le front de mer. Les hauteurs de Missiessy étant commandées par celle de Malbousquet, c'est finalement jusqu'à ce point que la nouvelle enceinte est envisagée. L'agrandissement ${ }^{4}$ sera alors de 100 ha (voir fig. $n^{\circ} 5$ ). Cette hypothèse réjouit la marine, qui constate que depuis Vauban, on a toujours prévu trop juste.

14 Si l'agrandissement de Castigneau est motivé par l'industrialisation de l'arsenal, celui de Missiessy aura pour but d'offrir un plan d'eau supplémentaire pour la flotte, ainsi qu'une réserve foncière pour le logement à terre des équipages et le cantonnement des troupes.

Adjugés en mars 1862, les travaux de creusement de la darse Missiessy s'étaleront jusqu'en 1872. À ce terme, l'arsenal est doté d'un plan d'eau supplémentaire de 19 ha, 
profond de $10 \mathrm{~m}$. Plus de deux millions de mètres cubes de déblais ont été extraits, dont une partie est réemployée pour former les terre-pleins des quais ou dans les ouvrages de fortifications. La nouvelle passe de Missiessy est dotée d'un pont tournant, sur lequel chemine la voie ferrée reliant l'arsenal au réseau P.L.M. depuis la gare de La Seyne. Trois nouvelles formes sont construites, entre 1876 et 1899, au fond de la darse.

À la fin du XIX ${ }^{e}$ siècle, l'arsenal de Toulon a presque atteint les limites qui sont aujourd'hui les siennes ${ }^{5}$ (fig. $\mathbf{n}^{\circ} \mathbf{6}$ ) (fig. $\mathbf{n}^{\circ} \mathbf{7}$ ). En quelques décennies, sa superficie a quadruplé, son outillage portuaire et ses moyens lui ont permis d'opérer la construction et le déploiement d'une marine nombreuse et moderne. Au tournant du $\mathrm{XX}^{\mathrm{e}}$ siècle, l'expansion de l'arsenal atteint son maximum. L'aménagement d'une école de pyrotechnie - entamée au milieu du XIX ${ }^{e}$ siècle - sur le rivage de Lagoubran, à l'extrémité occidentale de la rade, et la création de fosses de conservation des bois par immersion, confèrent au rivage du port de guerre une longueur de plus de $3000 \mathrm{~m}$ comptée depuis la darse vieille. Le dépôt pyrotechnique qui prend place entre l'école de pyrotechnie et la zone de Milhaud abrite les munitions et artifices de la flotte. Dans le secteur de Milhaud, des appontements sont construits durant la Première Guerre mondiale afin d'assurer le ravitaillement rapide des navires en charbon. En 1911, c'est près du cœur historique de l'arsenal qu'un terre-plein de quinze hectares est gagné sur la mer; il est destiné à recevoir quatre grandes formes de radoub, qui seront terminées en 1928, après interruption des travaux durant la guerre. À leur mise en service, ce sont les plus grandes formes au monde, capables de recevoir les plus grandes unités cuirassées qui seront lancées durant l'entre-deux guerres.

Figure 6

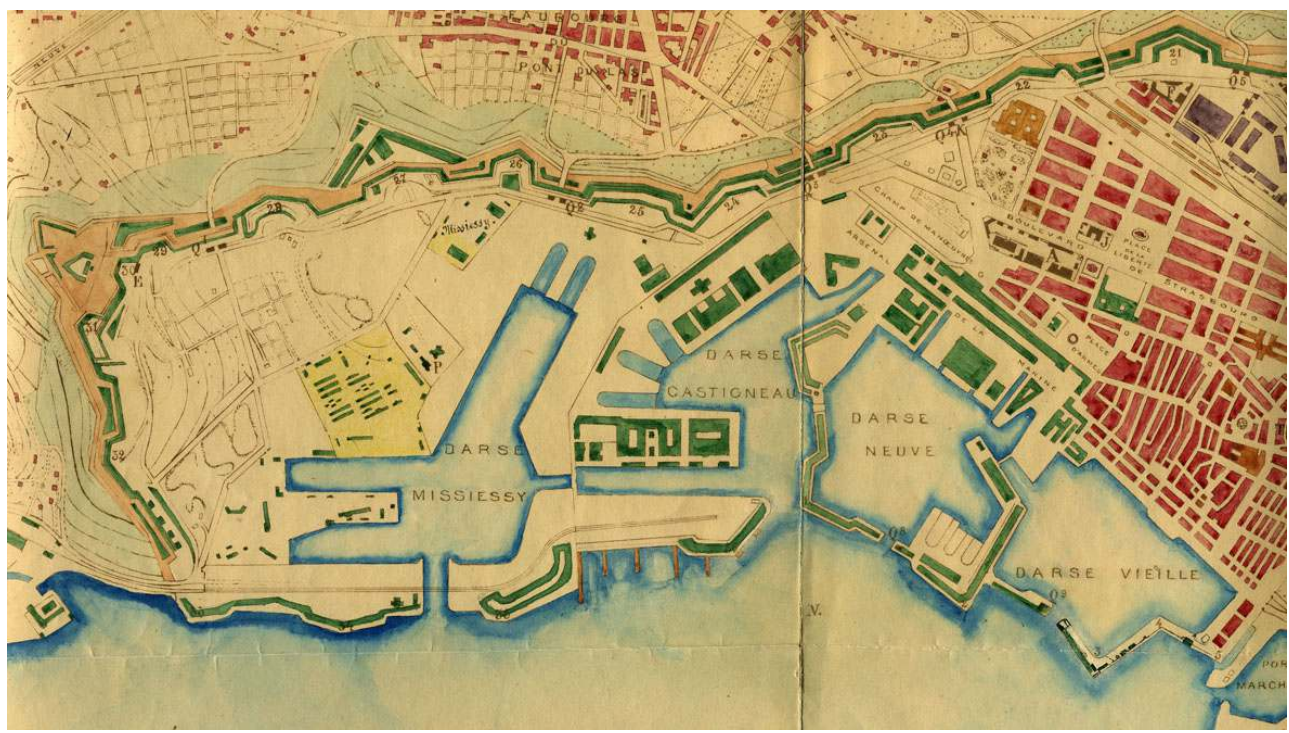

Plan de l'arsenal de Toulon en 1901 (Bibliothèque des Amis du vieux Toulon et de sa région). Phot. B. Cros. 


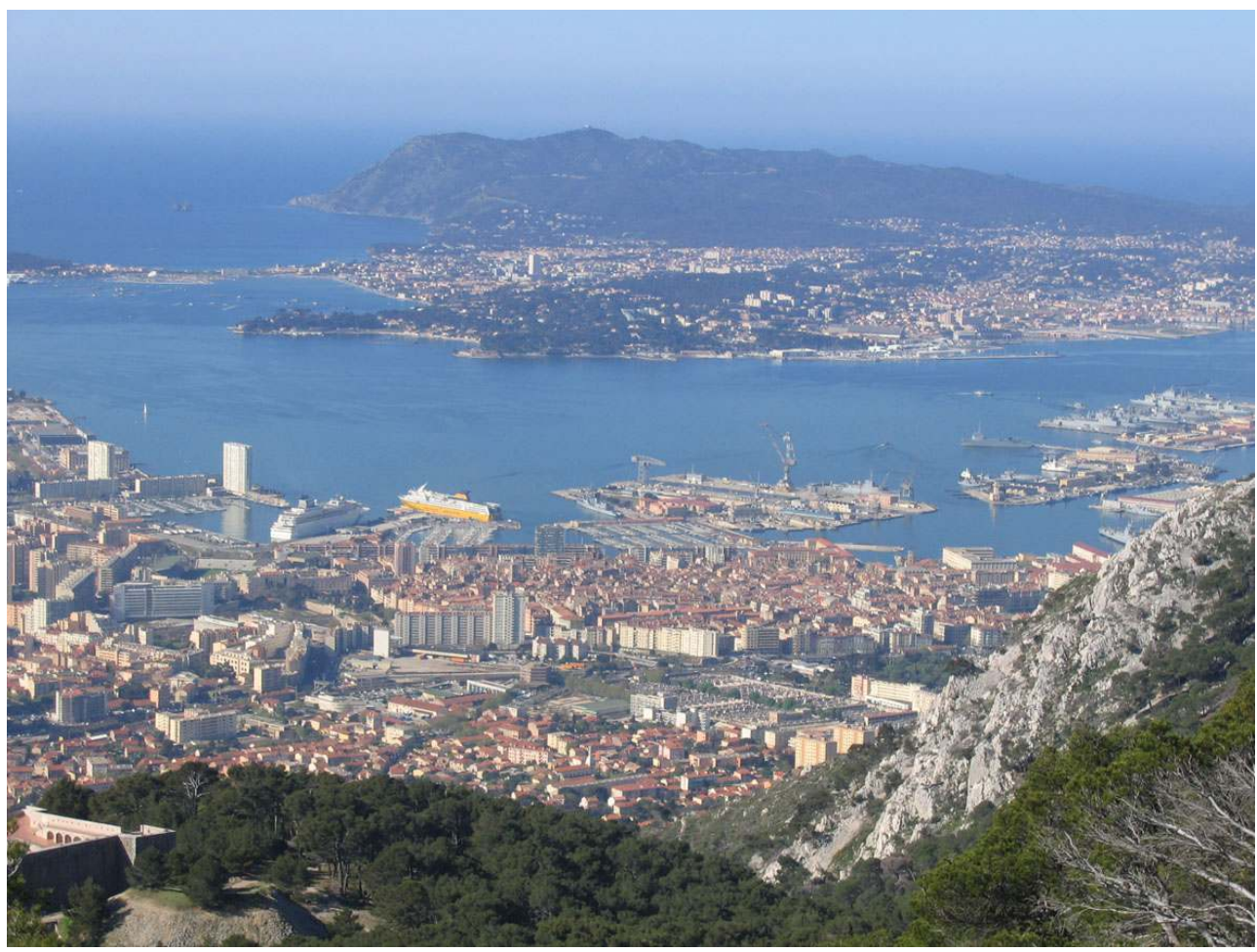

La rade de Toulon vue depuis le mont Faron. Phot. B. Cros.

\section{La marine, acteur de la politique d'aménagement urbain}

À la veille de la Seconde Guerre mondiale, le port de guerre est l'élément incontournable de l'espace toulonnais. Sur la rade, la marine occupe la quasi-totalité du rivage. En presqu'île de Saint-Mandrier, une base d'aéronautique maritime a été édifiée sur le rivage à proximité immédiate de l'hôpital maritime devenu école des mécaniciens de la marine en 1936. Le plan d'eau de la rade est lui-même à statut militaire. Son utilisation demeure libre à tout un chacun, mais sa sécurité et la surveillance de son exploitation relèvent de l'autorité maritime. Les forts et batteries qui couronnent chaque pointe ou cap, ou chaque point haut de quelque importance, sont désormais hérissés de canons anti-aériens ou de pièces anti-navires modernes. Après l'Occupation et la Libération, les installations immobilières et portuaires de la marine sont endommagées à plus de $50 \%$. Les nécessités de la reconstruction conduisent à une révision de l'urbanisation toulonnaise. L'avènement de l'automobile implique une reconfiguration du réseau viaire. La marine s'inscrit dans ces politiques d'intérêt général, tout en veillant aux intérêts de la défense nationale. Les «marges » de l'arsenal sont redessinées afin de faciliter la modernisation urbaine. La zone des remparts à l'ouest de l'arsenal voit la marine libérer une frange foncière destinée à permettre le tracé de la future autoroute venant de Marseille. Au contact du centre-ville, le secteur de la porte principale de l'arsenal est complètement requalifié pour améliorer la circulation automobile et aérer la trame du bâti urbain. Un temps envisagée, la cession à la Ville de l'arsenal du Mourillon est reportée sine die, à cause du coût des relogements dans l'arsenal des services qui seraient touchés. Ces 
remises en question d'ordre foncier sont amorcées au lendemain de la Libération et se poursuivront sur une période de trente ans.

En dépit des destructions des guerres, la marine à Toulon est toujours détentrice d'un patrimoine immobilier et portuaire qui traduit l'ancienneté et l'étendue de sa présence autour de la rade. L'arsenal, désormais qualifié de Base navale, conserve des témoins de sa fondation (ouvrage d'artillerie de l'Angle Robert, des années 1630). La période de l'Ancien régime est marquée par des ouvrages exceptionnels, comme la corderie ${ }^{6}$ (1686-vers 1700$)$, la goudronnerie et le magasin au goudron (vers 1685), le bâtiment de l'horloge (1776), la boulangerie (vers 1780) et la forme Groignard ${ }^{7}(1778)$ toujours opérationnelle (fig. $\left.\mathbf{n}^{\circ} \mathbf{8}\right)$, l'ancien magasin à poudre de Milhaud $(1697)^{8}$ (fig. $\left.\mathbf{n}^{\circ} \mathbf{9}\right)$, (fig. $\left.\mathbf{n}^{\circ} \mathbf{1 0}\right)$, (fig. $\left.\mathbf{n}^{\circ} \mathbf{1 1}\right)$.

Figure 8

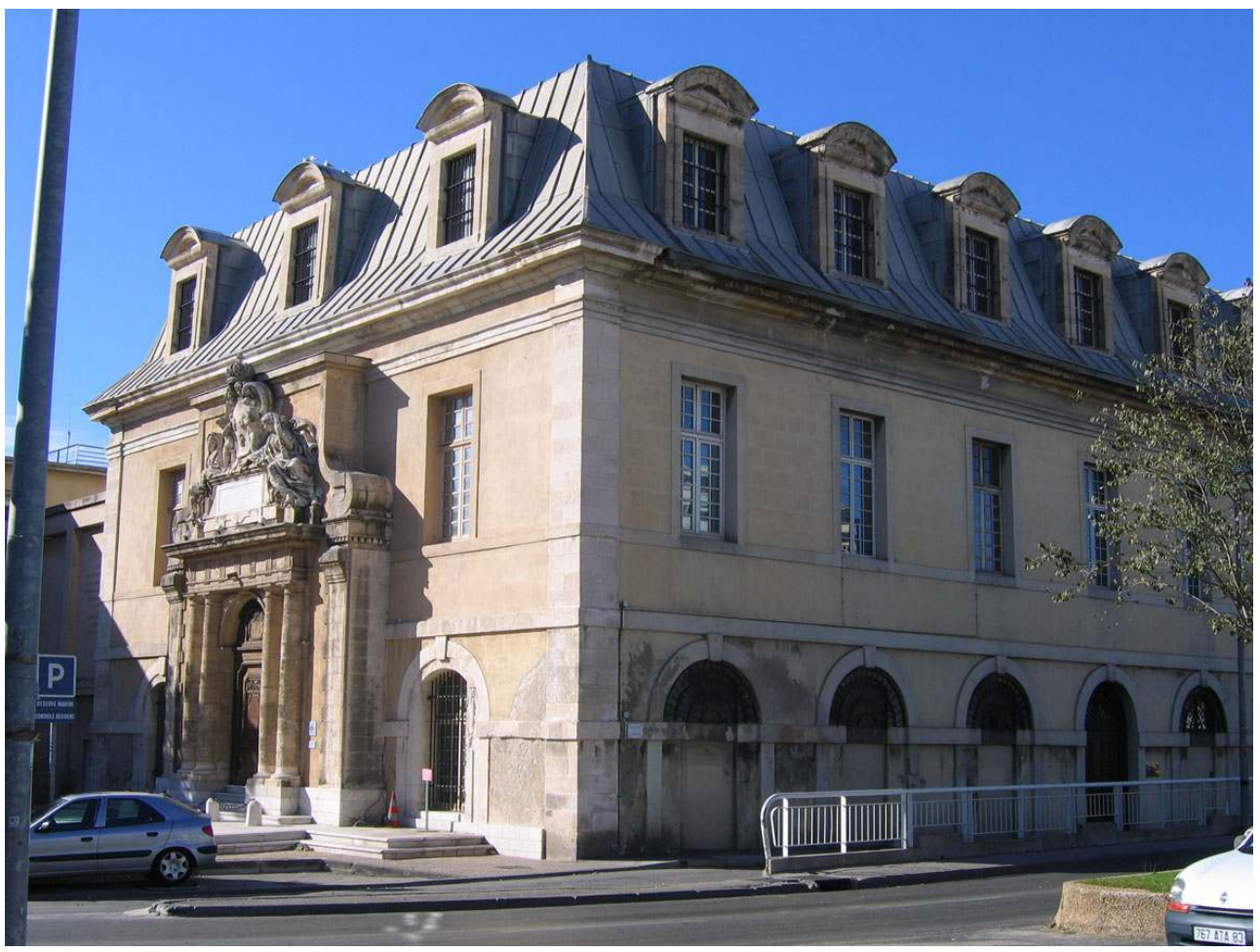

Pavillon Est de la corderie. Phot. B. Cros. 
Figure 9

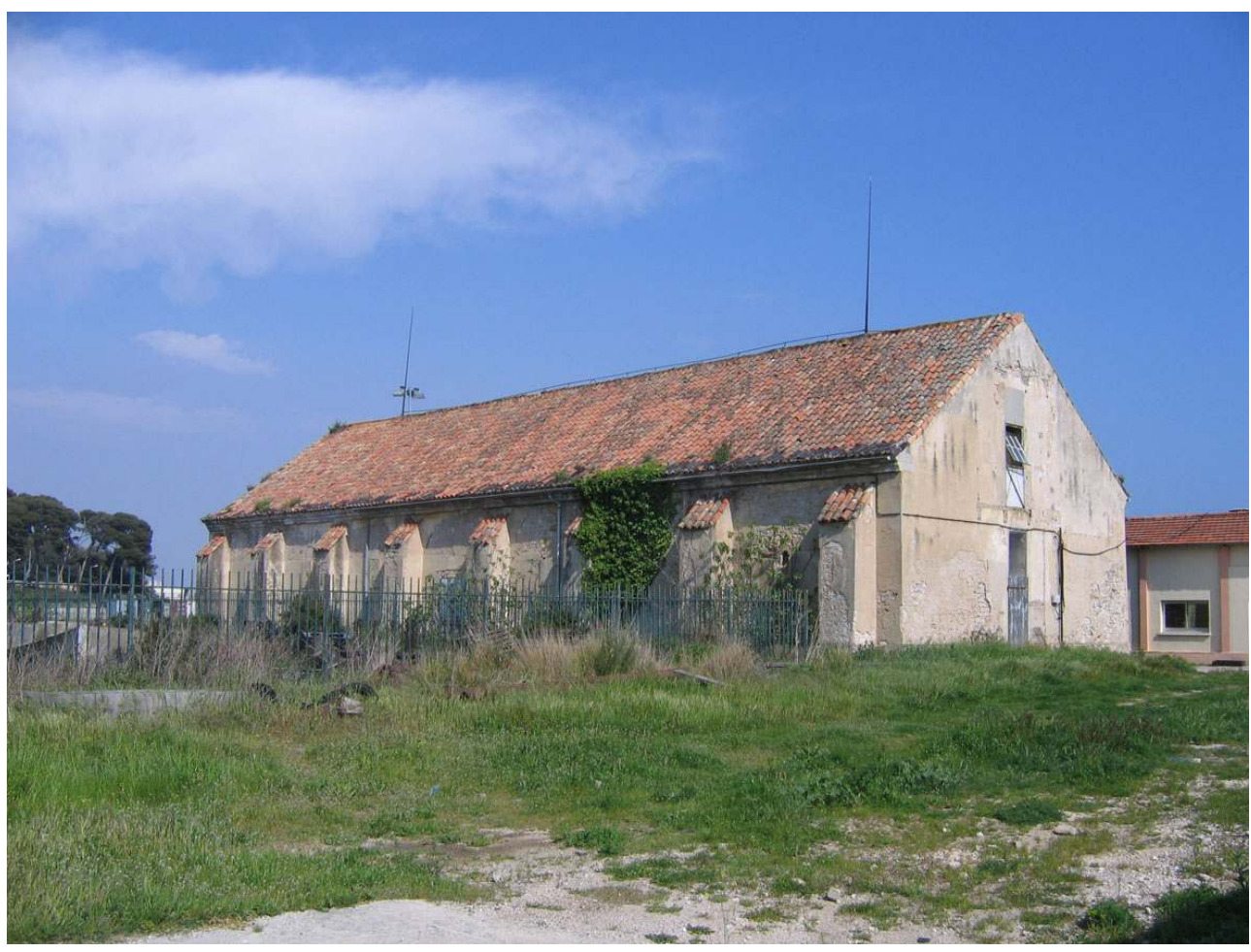

L'ancien magasin à poudre de Milhaud. Phot. B. Cros.

\section{Figure 10}

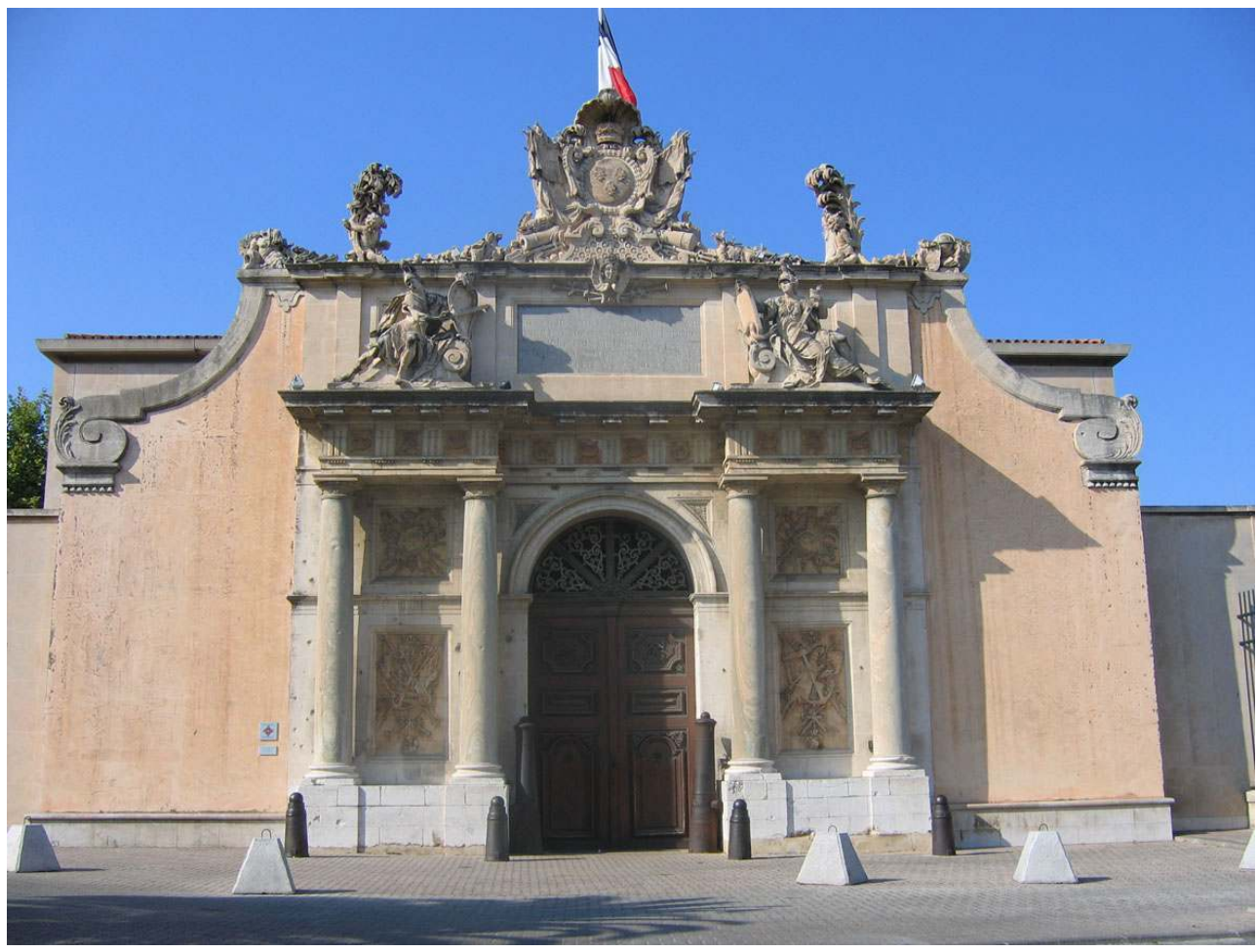

L'ancienne porte monumentale de l'arsenal. Phot. B. Cros. 


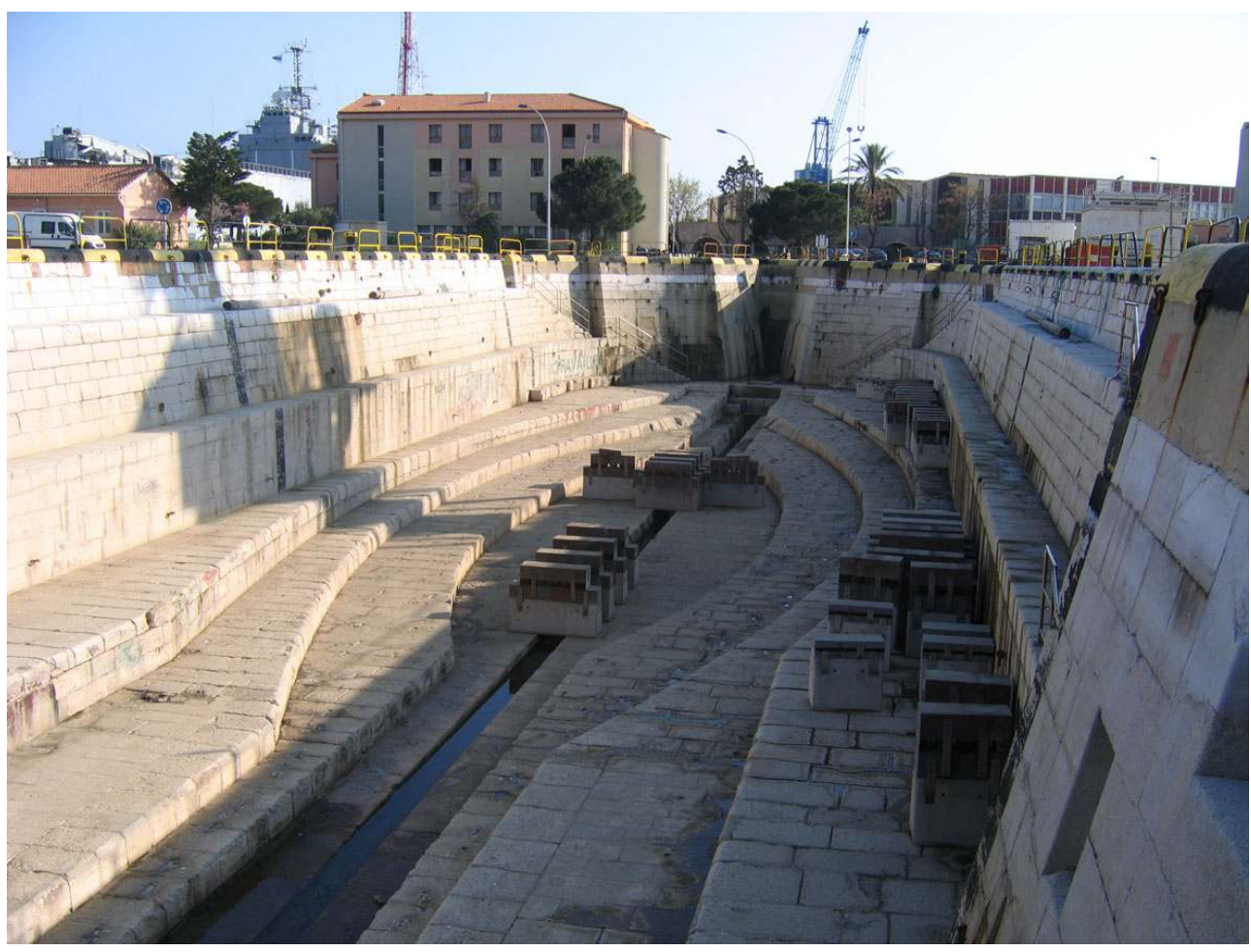

Bassin de radoub « Groignard ». Phot. B. Cros.

19 L'œuvre des forçats est bien visible, mais ignorée, avec deux petits bassins Vauban et l'ancien magasin général. Les remparts de Malbousquet, dernière enceinte bastionnée édifiée en France à la veille de la guerre de 1870, constituent un ensemble magistral et méconnu. Sur la presqu'île de Saint-Mandrier, l'ancien hôpital est désormais partie intégrante du Centre d'instruction naval (CIN), la plus importante école de la marine. De nombreux ouvrages fortifiés font toujours partie du patrimoine de la marine, nonobstant les multiples cessions opérées au profit des collectivités locales depuis quatre décennies. Ce sont ainsi une dizaine de forts et une douzaine de batteries de côte que l'histoire nous a transmis, dans un état de conservation favorisé par la clémence du climat et des effets relativement peu destructeurs des guerres.

\section{Conservation et protection du patrimoine}

\section{Identification et recensement}

20 La reconnaissance officielle de la qualité historique et architecturale du patrimoine militaire est relativement récente en France. Elle est longtemps demeurée embryonnaire s'agissant du patrimoine militaire à Toulon. Quelques ouvrages de défense côtière bénéficient d'une protection au titre des Monuments historiques : la Grosse tour, ou tour royale classée en 1947, le fort Saint Louis et le fort de Balaguier, inscrits en 1948 et en 1975. Du côté des fortifications terrestres, la porte Malbousquet a été inscrite en 1989, au terme d'une négociation menée avec le ministère de la Défense afin d'empêcher sa destruction programmée. La prise de conscience de la notion de patrimoine militaire se fait jour au début des années 1990. En 1992, la marine nationale crée la Commission du 
patrimoine de la marine ${ }^{9}$ (CPM). Dans ce mouvement, le service des travaux immobiliers et maritimes de la marine met sur pied une mission patrimoine, chargée d'œuvrer de concert avec la CPM. Sa première tâche consiste à identifier le patrimoine immobilier de la marine « digne d'intérêt » : cette notion a pour objet de détecter le patrimoine qui, bien que non protégé au titre des Monuments historiques, serait susceptible de bénéficier de la même attention dans sa conservation, son exploitation et son éventuelle mise en valeur. S'agissant de la rade de Toulon, le patrimoine portuaire est passé au peigne fin, tout autant que le patrimoine fortifié affecté à la marine, qui a « hérité » de l'armée de terre la totalité de ses ouvrages fortifiés entre le milieu du XIXe siècle et l'entre-deux guerres. Le résultat, éloquent, constitue une véritable découverte, car c'est la première fois que ce patrimoine, pourtant quotidiennement occupé et utilisé dans sa très grande majorité, fait l'objet d'une mise en perspective à visée culturelle. L'histoire sous-jacente des ouvrages concernés avait fini par tomber dans les oubliettes de la mémoire collective. Le tableau qui suit présente ainsi la synthèse des ouvrages portuaires et associés de l'arsenal recensés dans ces conditions :

\begin{tabular}{|c|c|c|c|}
\hline Ouvrage & Datation & Protection & Observations \\
\hline Fortin de l'angle Robert & v. 1638 & & Ancienne terrasse d'artillerie \\
\hline Bâtiment de l'horloge & 1776 & & $\begin{array}{l}\text { Construit par l'ingénieur Verguin, } \\
\text { ancien membre de l'expédition La } \\
\text { Condamine en Amérique méridionale }\end{array}$ \\
\hline $\begin{array}{l}\text { Quais de la darse de } \\
\text { l'horloge }\end{array}$ & $\mathrm{XVIII}{ }^{\mathrm{e}}$ & & $\begin{array}{l}\text { Ancien quai d'honneur de l'arsenal. } \\
\text { Orné de masques de lion en bronze } \\
\text { fondus vers } 1685 \text { à Toulon }\end{array}$ \\
\hline Bassin Groignard & 1778 & & $\begin{array}{l}\text { Premier bassin de radoub français en } \\
\text { Méditerranée. Pratiquement non } \\
\text { modifié depuis sa construction }\end{array}$ \\
\hline Petit bassin Vauban 2 & 1838 & & $\begin{array}{l}\text { Entièrement construit par les forçats. } \\
\text { Première expérience de bétonnage à la } \\
\text { mer en grande masse }\end{array}$ \\
\hline Petit bassin Vauban 3 & 1847 & & $\begin{array}{l}\text { Entièrement construit par les forçats. } \\
\text { Pratiquement non modifié depuis sa } \\
\text { construction }\end{array}$ \\
\hline $\begin{array}{l}\text { Ancien magasin } \\
\text { général }\end{array}$ & $1803-1827$ & & $\begin{array}{l}\text { Construit en partie par les forçats. } \\
\text { Partiellement détruit durant la guerre }\end{array}$ \\
\hline $\begin{array}{l}\text { Aile nord de l'ancienne } \\
\text { boulangerie }\end{array}$ & $\begin{array}{l}\text { Entre } 1750 \\
\text { et } 1780\end{array}$ & & $\begin{array}{l}\text { Vestige de la seconde aile de la } \\
\text { boulangerie, construite par Verguin et } \\
\text { achevée par Verrier }\end{array}$ \\
\hline $\begin{array}{l}\text { Centre Malbousquet } \\
\text { (ancien «Dépôt des } \\
\text { équipages ») }\end{array}$ & 1907 & & $\begin{array}{l}\text { Casernes de la marine construites } \\
\text { selon un style Napoléon III }\end{array}$ \\
\hline
\end{tabular}




\begin{tabular}{|c|c|c|c|}
\hline $\begin{array}{l}\text { Fortifications de } \\
\text { Malbousquet }\end{array}$ & 1870 & $\begin{array}{l}\text { Porte } \\
\text { Malbousquet, } \\
\text { ISMH }\end{array}$ & $\begin{array}{l}\text { Front fortifié long de } 1900 \mathrm{~m} \text {, avec } \\
\text { casemates de fusillades et extérieurs. } \\
\text { Porte, poternes et magasins divers }\end{array}$ \\
\hline $\begin{array}{l}\text { Magasin à poudre de } \\
\text { Milhaud }\end{array}$ & 1697 & & $\begin{array}{l}\text { Magasin à deux niveaux voûtés et huit } \\
\text { contreforts. Pratiquement dans son } \\
\text { état d'origine }\end{array}$ \\
\hline $\begin{array}{l}\text { Ancien corps de garde } \\
\text { de Missiessy }\end{array}$ & v. 1862 & & $\begin{array}{l}\text { Ancien poste de garde du pont mobile } \\
\text { de la passe Missiessy }\end{array}$ \\
\hline Ancienne corderie & $\begin{array}{l}1685- \\
\text { v. } 1700\end{array}$ & & $\begin{array}{l}\text { Aile à trois travées encadrée de deux } \\
\text { pavillons. Longue de } 400 \mathrm{~m} \text {, dont } \\
\text { interruption de } 45 \mathrm{~m} \text { par faits } \\
\text { d'incendie }\end{array}$ \\
\hline $\begin{array}{l}\text { Ancien magasin au } \\
\text { goudron }\end{array}$ & 1685 & & \\
\hline Ancienne goudronnerie & 1685 & & \\
\hline $\begin{array}{l}\text { Ancienne porte } \\
\text { principale de l'arsenal }\end{array}$ & 1738 & MH & $\begin{array}{l}\text { Déplacée en 1976. Plaquée en façade } \\
\text { du musée de la marine }\end{array}$ \\
\hline $\begin{array}{l}\text { Porte de l'ancien } \\
\text { séminaire de la marine }\end{array}$ & 1690 & MH & $\begin{array}{l}\text { Déplacée en } 1911 \text { et } 1976 . \\
\text { Actuellement plaquée en pignon est de } \\
\text { la corderie }\end{array}$ \\
\hline $\begin{array}{l}\text { Vestige d'enceinte de la } \\
\text { darse neuve }\end{array}$ & v. 1693 & & $\begin{array}{l}\text { Tronçon de rempart sans son parapet, } \\
\text { long de } 25 \mathrm{~m}\end{array}$ \\
\hline
\end{tabular}

Ce recensement démontrait que, contrairement à des assertions fréquemment répandues, l'arsenal de Toulon dispose encore de témoins significatifs du siècle de Louis XIV. Une des conséquences directes de ce travail d'approche dans un contexte d'ensemble a probablement été la sauvegarde de l'ancien magasin à poudre de Milhaud: unique exemplaire de cette époque encore présent dans un port militaire, il est situé dans une zone de l'arsenal à caractère opérationnel. Au milieu des années 1990, la décision de principe a été prise de le démolir. Le recensement du patrimoine conduit peu de temps auparavant a permis de prendre conscience de sa valeur et a motivé la recherche de solution alternative à sa destruction. 


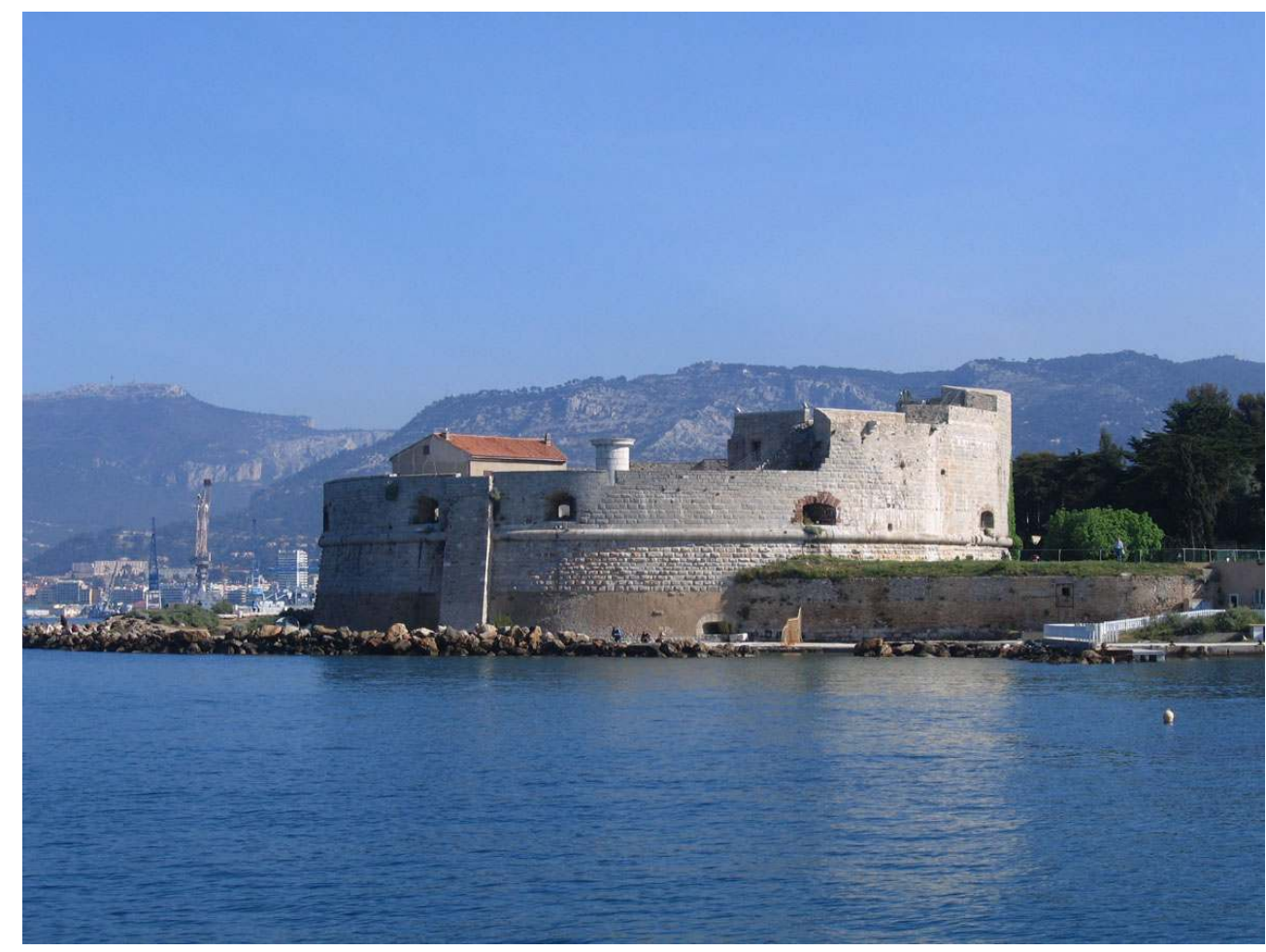

La Grosse tour ou tour royale. Phot. B. Cros.

Le recensement des ouvrages fortifiés situés en périphérie de la rade de Toulon illustre très bien l'histoire de la fortification - et particulièrement de la défense des côtes - sur un éventail chronologique s'étendant du début du XVI ${ }^{\mathrm{e}}$ siècle à la veille de la Seconde Guerre mondiale. En matière de défense côtière, chaque "strate" de l'architecture militaire française est bien représentée. La tour royale, ou Grosse tour de Toulon (1524) fait ainsi écho au château d'If ou à la tour de Sainte-Agathe, tours à canons édifiées sous le règne de François $\mathrm{I}^{\mathrm{er}}$ avant même que Toulon ne fût port de guerre (fig. $\left.\mathbf{n}^{\circ} \mathbf{1 2}\right)$. Le fort de Balaguier (1636) est le maillon toulonnais (pris au sens large, puisque édifié à La Seyne-sur-Mer) du système défensif élaboré par Richelieu sur les côtes provençales face aux ambitions espagnoles. La construction du grand arsenal sous le règne de Louis XIV implique un renforcement des défenses côtières, visible aujourd'hui à travers les forts de l'Eguillette et Saint-Louis ${ }^{10}$. La remise à niveau de la défense des côtes après 1840 est bien lisible à travers la modernisation du fort de l'Eguillette, mais aussi avec la construction d'un ensemble de batteries de plan normalisé ${ }^{11}$ encore présentes de Sanary à Carqueiranne ${ }^{12}$. La remise en cause du système défensif par les progrès de l'artillerie dans les années 1860 aboutit, en 1873, à l'élaboration d'un nouveau plan de défense. Sa mise en application, à partir de 1876, inscrit dans le paysage toute une série d'ouvrages aux lignes épurées, dominant la mer afin d'accroître son efficacité contre les navires de guerre, tout en s'en préservant plus efficacement. Plus de quinze ouvrages en témoignent encore maintenant, entre Six-Fours et Carqueiranne. L'étape la plus récente correspond au programme de défense du littoral entrepris par la marine durant l'entre-deux guerres. D'ampleur plus limitée, elle n'en est pas moins spectaculaire. Les dessous de la batterie de $340 \mathrm{~mm}$ de Saint-Mandrier, d'une portée de $40 \mathrm{~km}$, sont toujours bien conservés et d'une portée historique exceptionnelle. Du côté de la défense terrestre, la ligne de forts édifiée au XVIII 
e siècle présente des témoins diversifiés : imposant fort Lamalgue aux nombreux dehors éloquents, débris épars de la redoute Sainte-Catherine, frappée de plein fouet par la construction de hautes tours d'habitation à la fin des années 1960, campagnarde redoute des Pomets intelligemment occupée par ses propriétaires privés. Le Mont Faron porte les témoins bien conservés de son occupation militaire permanente entreprise en 1840. Les points hauts environnant Toulon portent enfin les forts détachés du système Séré de Rivières, qui se développe le long d'une ligne de $35 \mathrm{~km}$, allant de Six-Fours à Carqueiranne en passant par Sanary et Évenos. (fig. $\left.\mathbf{n}^{\circ}{ }^{13}\right)$ (fig. $\left.\mathbf{n}^{\circ}{ }^{14}\right)\left(\right.$ fig. $\left.^{\circ}{ }^{\circ} 15\right)\left(\right.$ fig. $\left.^{\circ}{ }^{\circ} 16\right)$

\section{Figure 13}

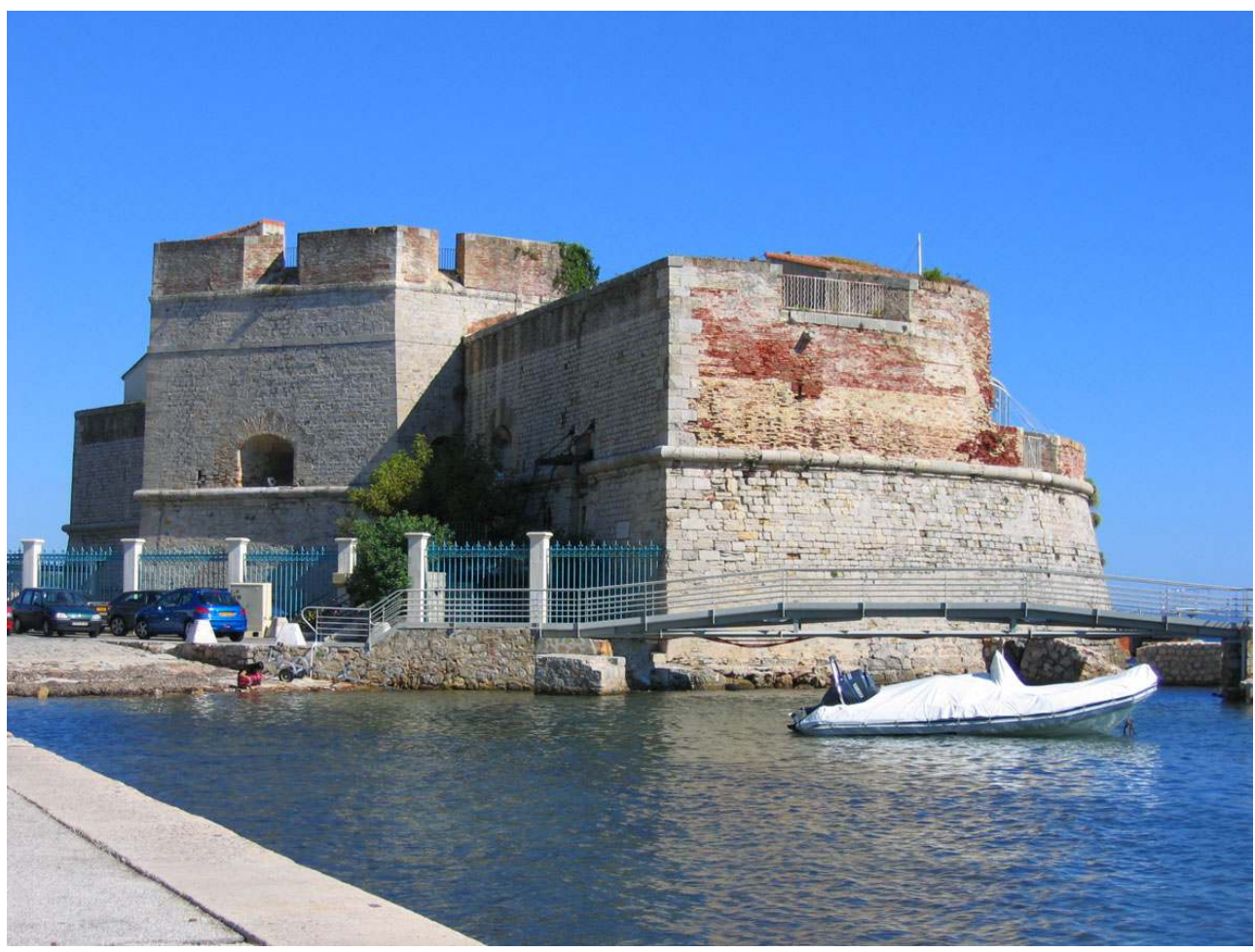

Le fort Saint Louis, ou fort des Vignettes. Phot. B. Cros. 


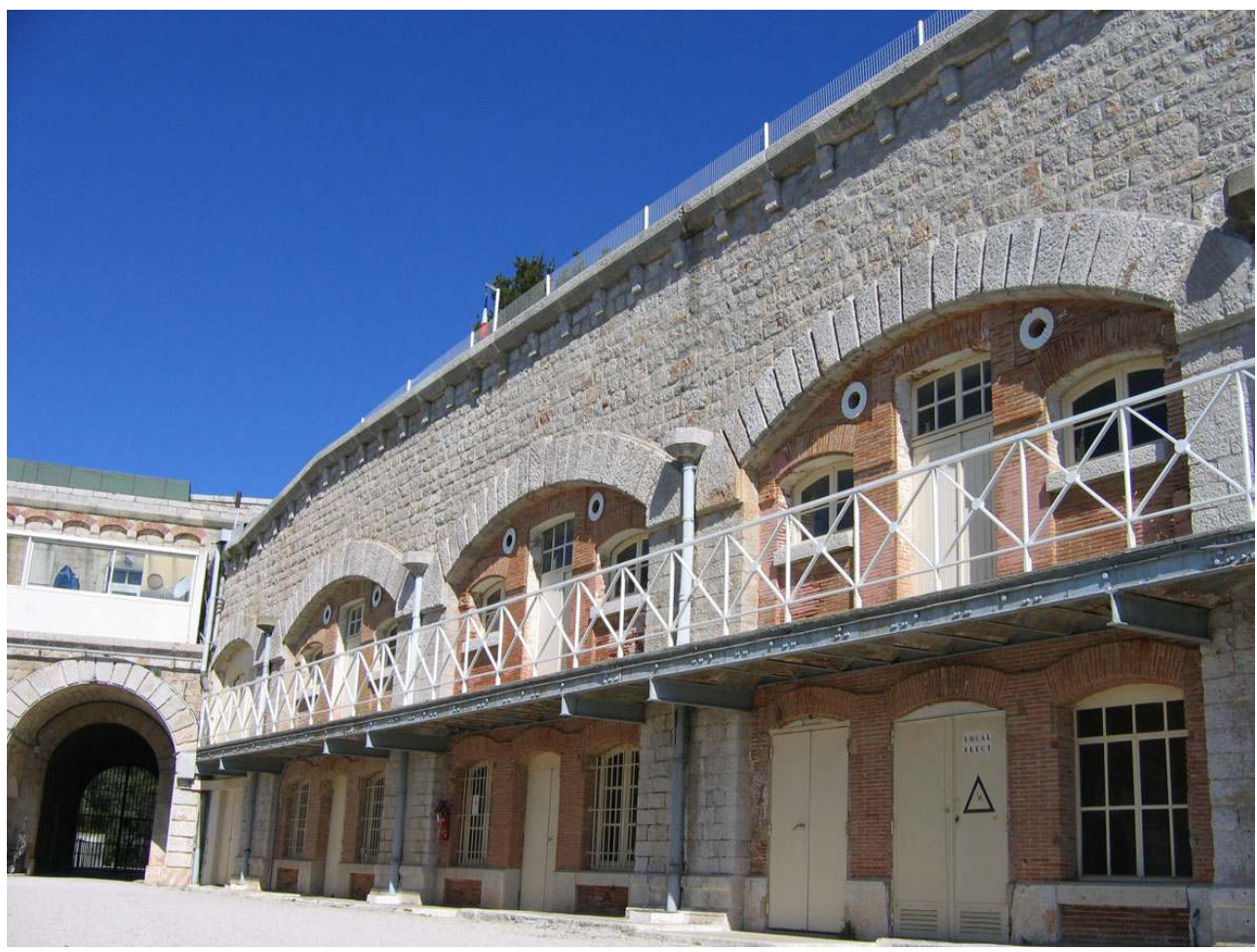

Caserne du fort de la Croix-Faron. Phot. B. Cros.

\section{Figure 15}

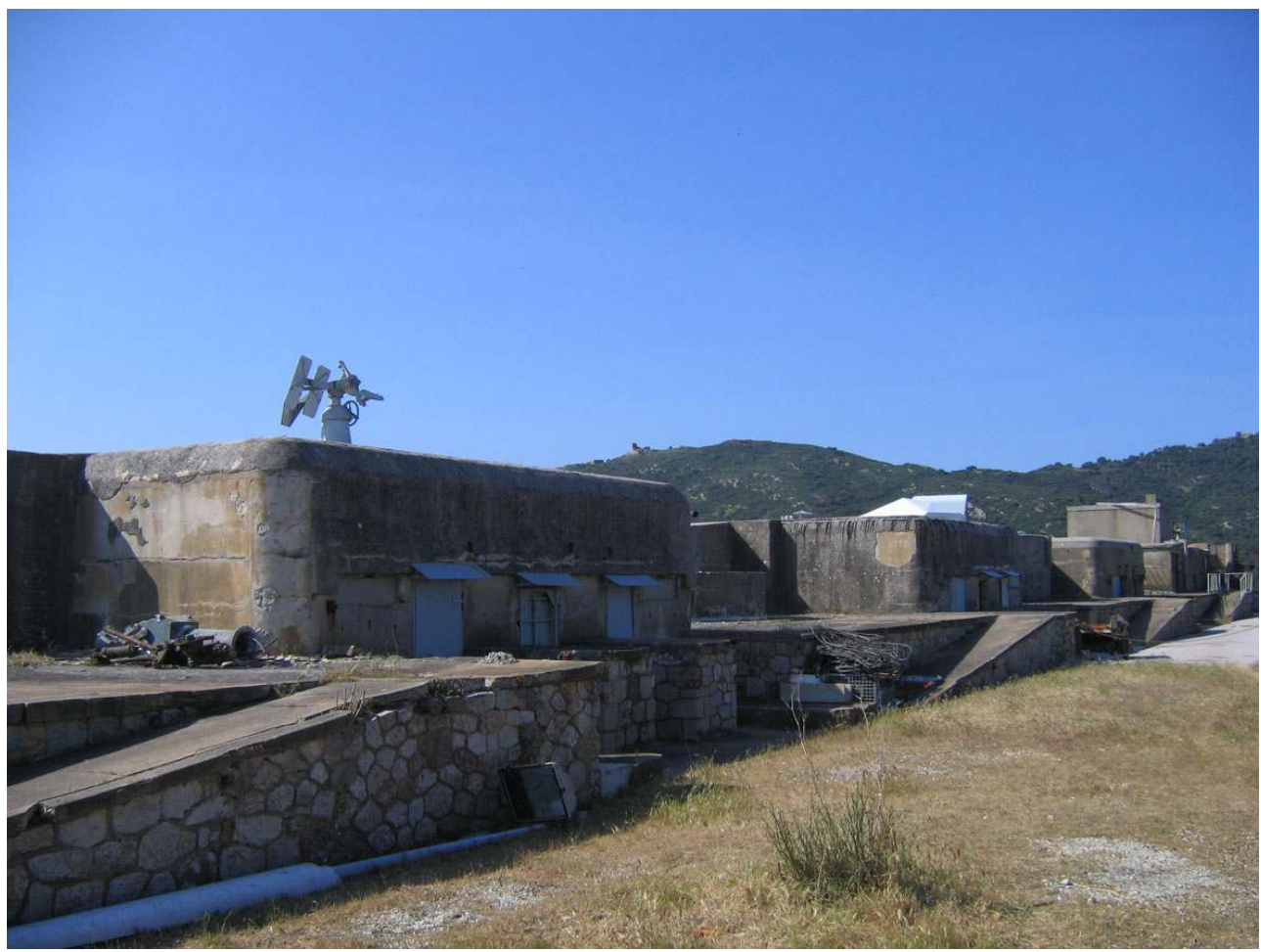

La batterie de Peyras. Phot. B. Cros. 
Figure 16

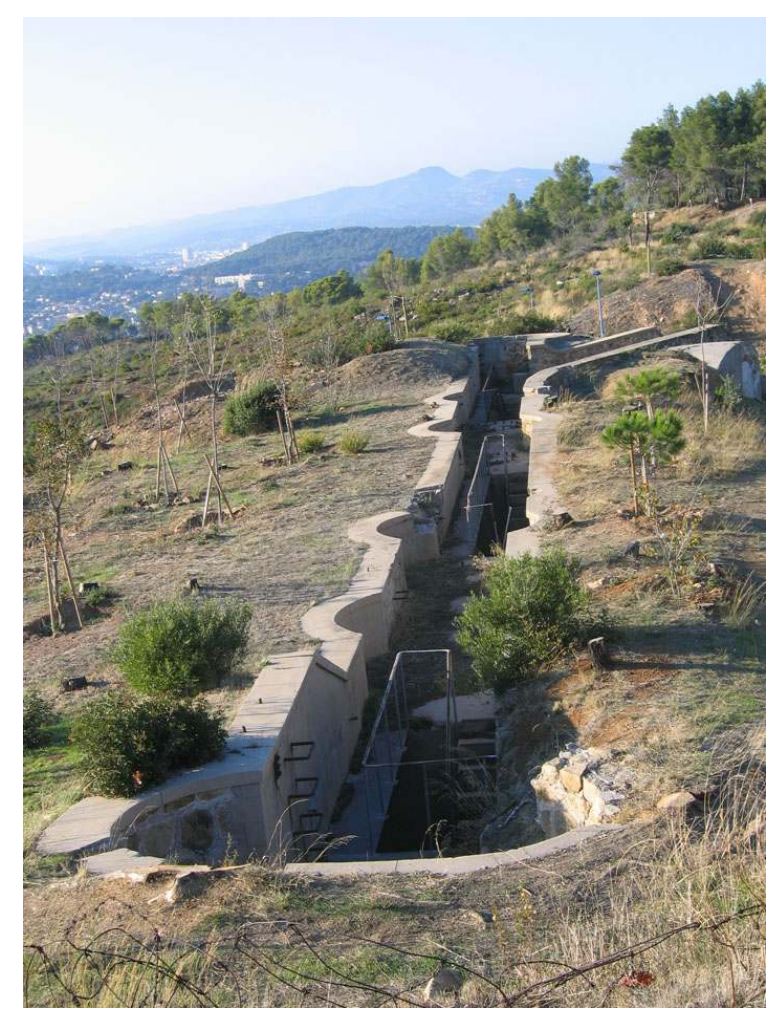

Batterie haute du Lazaret. Phot. B. Cros.

Ce sont ainsi une petite cinquantaine d'ouvrages fortifiés qui s'égrènent sur les rivages et points hauts de l'aire toulonnaise, dont les trois quarts sont toujours affectés à la Défense/Marine. Parvenus jusqu'à nous en assez bon état de conservation, en dépit des combats qui ont marqué l'histoire locale, tous ces ouvrages constituent une sorte de «musée éclaté » de la fortification. Et pourtant, une faible poignée d'entre eux sont protégés au titre des Monuments historiques : un fort côtier est classé, deux autres sont inscrits. Un fort terrestre est inscrit.

\section{Palette d'actions}

Les travaux de restauration conduits dans la décennie 1990-2000 ont mobilisé au mieux les ressources offertes par le protocole Culture-Défense signé en 1983 (renouvelé depuis en 1990 et septembre 2005), applicable en pratique aux seuls édifices classés. La porte de l'ancien séminaire fut restaurée par ce biais en 1994, tandis que la Grosse tour se voyait inscrite dans un programme pluriannuel de travaux. Ces travaux étaient guidés par un double souci : assainir le monument pour garantir sa pérennité, permettre son ouverture au public. Les interventions menées sur le gros-œuvre ont consisté à étancher les parements exposés aux intempéries, régénérer la masse des maçonneries imbibées par des siècles de pluies de sud-est. En parallèle, les terrasses supérieures étaient étanchées, avec dépose et repose des dalles de pavement. Les embrasures des casemates à canons étaient rouvertes, ainsi que les exutoires de fumées de tir. Ces différents travaux ont permis de régénérer les maçonneries et d'assainir durablement l'ouvrage. La restauration des grands et petits escaliers, combinée avec des travaux d'éclairage et de sécurisation 
financés directement par la Marine, a permis une réouverture complète du monument au public, ainsi mis en conformité avec les règles ERP ${ }^{13}$. Cédé à la Ville en 2006, le monument était pour lors muni des meilleures dispositions, même si beaucoup reste à faire pour le mettre en situation d'une exploitation digne de ses qualités intrinsèques (fig. $\mathbf{n}^{\circ} \mathbf{1 7}$ ).

Figure 17

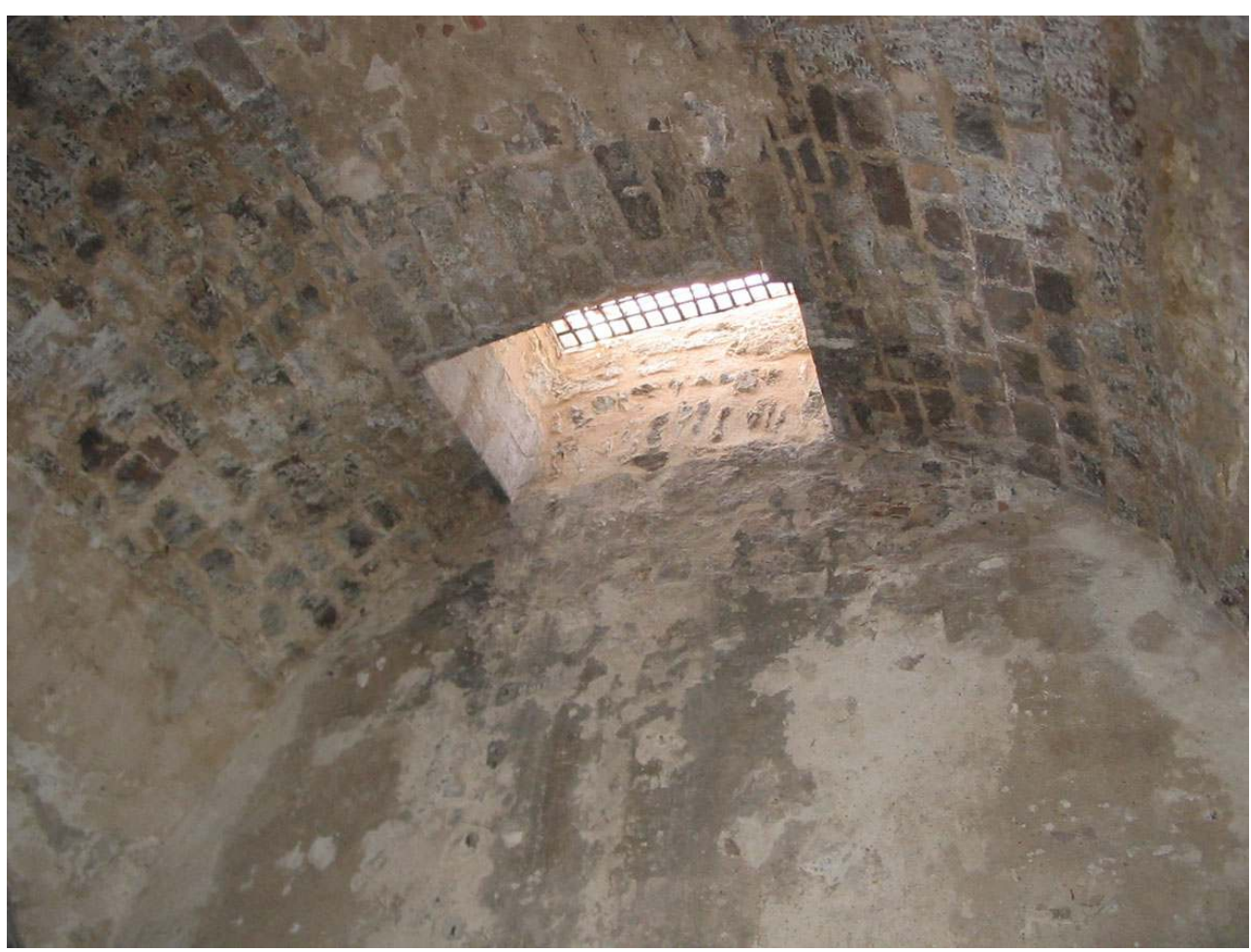

Tour royale. Ventilation haute de casemate. Phot. B. Cros

La conservation du patrimoine n'est pas la mission première de la marine nationale. Mais, pour ce qui concerne le patrimoine utilisé pour les missions de la marine, le recensement et la documentation du patrimoine "digne d'intérêt» ont pour but de sensibiliser les intervenants aux soins qui doivent accompagner les "gestes » affectant ces ouvrages. Choix du parti architectural pour les interventions de grande ampleur, appréhension et respect du contexte architectural existant pour les interventions de petite ampleur, sont autant d'axes de sensibilisation présentés aux décideurs, occupants et maîtres d'œuvre concernés. Il s'agit, bien entendu, d'une démarche de longue haleine, d'autant plus nécessaire dans la durée que les personnels concernés sont soumis à un turnover constant. Qu'il soit patrimoine "digne d'intérêt» ou bien monument inscrit à l'inventaire supplémentaire des Monuments historiques, le patrimoine à caractère historique de la marine ne peut être conservé qu'en mobilisant ses propres crédits d'infrastructure. Dans une période où l'abondance n'est pas de mise, des priorités doivent parfois être arbitrées entre nécessités opérationnelles (voire simple maintien en condition d'infrastructures vieillissantes) et conservation de monuments historiques inscrits. Il en va ainsi pour la remarquable chapelle de Saint-Mandrier (ISMH), dont l'étude de restauration a pu être menée à bien après un financement plusieurs fois reporté. La réalisation des travaux de stricte conservation devra probablement patienter encore quelque peu (fig. $\left.\mathbf{n}^{\circ} \mathbf{1 8}\right)$. 
Figure 18

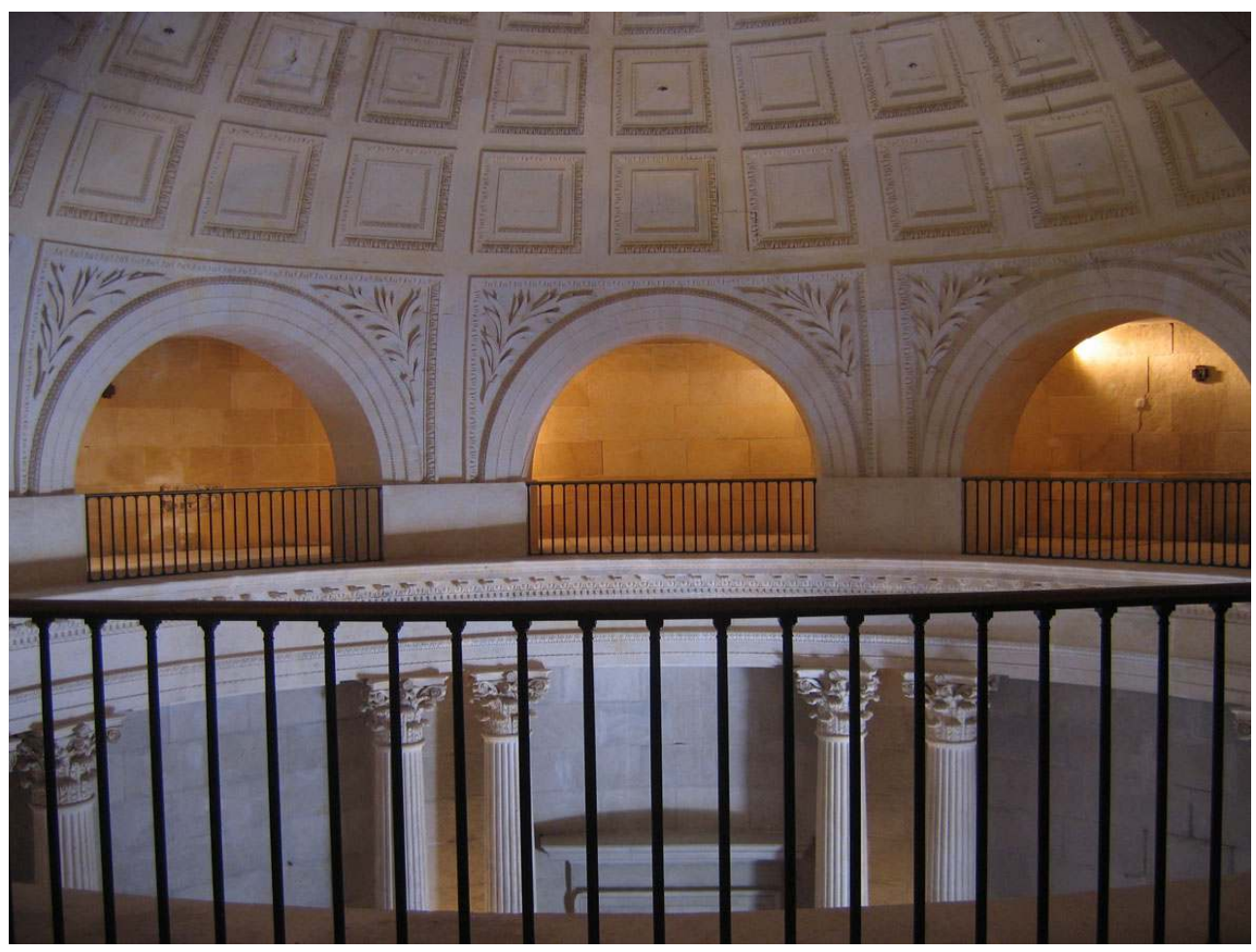

Intérieur de la chapelle de Saint-Mandrier. Phot. B. Cros.

La base navale de Toulon constitue un des sites opérationnels majeurs de la défense française. Port base de la plus grande partie de la flotte, elle constitue également un des points principaux à partir desquels sont projetées les forces françaises en opérations extérieures. Pour améliorer ses capacités de desserte par voie routière, il fut envisagé, dans les années 1985, de créer un passage sans limitation de gabarit en lieu et place de la porte Malbousquet, témoin des fortifications 1860-70 de l'arsenal. Pour empêcher une telle destruction, le ministère de la Culture proposa alors de classer l'édifice. Au terme de négociations interministérielles, la porte fut inscrite à l'inventaire supplémentaire des Monuments historiques, le projet d'amélioration de la circulation amendé et un financement de la Culture vint abonder le budget de la Défense. Aujourd'hui restaurée, la porte a en outre bénéficié d'aménagements complémentaires dissimulés dans la masse du rempart. Sa desserte par voie autoroutière en fait en effet le point d'accès principal à la base navale. L'organisme chargé d'administrer les autorisations d'accès est désormais installé dans les volumes créés à proximité immédiate sans modifier son aspect extérieur.

Pour des ouvrages sans utilisation, du fait de leur état, mais susceptibles d'une exploitation subordonnée à leur remise en état, il a pu être recouru aux moyens associatifs. Le cas du fort de l'Eguillette est particulièrement emblématique à cet égard. Construit sous Louis XIV pour compléter le contrôle sur l'entrée de la petite rade, l'ouvrage a été remanié largement en 1846 et 1859. En 1996, la marine nationale a engagé un partenariat avec une association animant des chantiers d'insertion ${ }^{14}$ et qui s'était auparavant illustrée en restaurant le fort Faron, puis en y conduisant des activités à caractère pédagogique. Après un minutieux travail documentaire et d'interprétation de l'édifice, le parti général des travaux a été défini, visant à restaurer l'ouvrage dans un état aussi conforme que possible à celui de 1859, correspondant à « l'apogée » de sa vocation 
défensive avant son déclassement en 1877 et les enlaidissements purement fonctionnels $\mathrm{du} \mathrm{XX}^{\mathrm{e}}$ siècle, sans aucun rapport avec son caractère défensif ${ }^{15}$. Réalisés par tranches annuelles depuis 1997, les travaux ont atteint en quelques années un degré d'avancement propice à l'ouverture du fort au public. De façon limitée aux journées du patrimoine pour commencer, puis sur la durée de la belle saison depuis quelques années (expositions, spectacles de théâtre ou de musique, forums, mariages). La complète restauration du fort devrait être atteinte d'ici cinq années. La marine nationale y contribue en effectuant les recherches documentaires et en fournissant ses directives en maîtrise d'ouvrage. La conduite d'opération est effectuée par le service d'infrastructure de la défense, qui a procédé aux études documentaires. Le financement des matériaux est assuré par la marine nationale. L'association prend en charge le financement des chantiers d'insertion, en provenance des collectivités territoriales ${ }^{16}$ (fig. $\left.\mathbf{n}^{\circ} 19\right)$, (fig. $\left.\mathbf{n}^{\circ} \mathbf{2 0}\right)$.

Figure 19

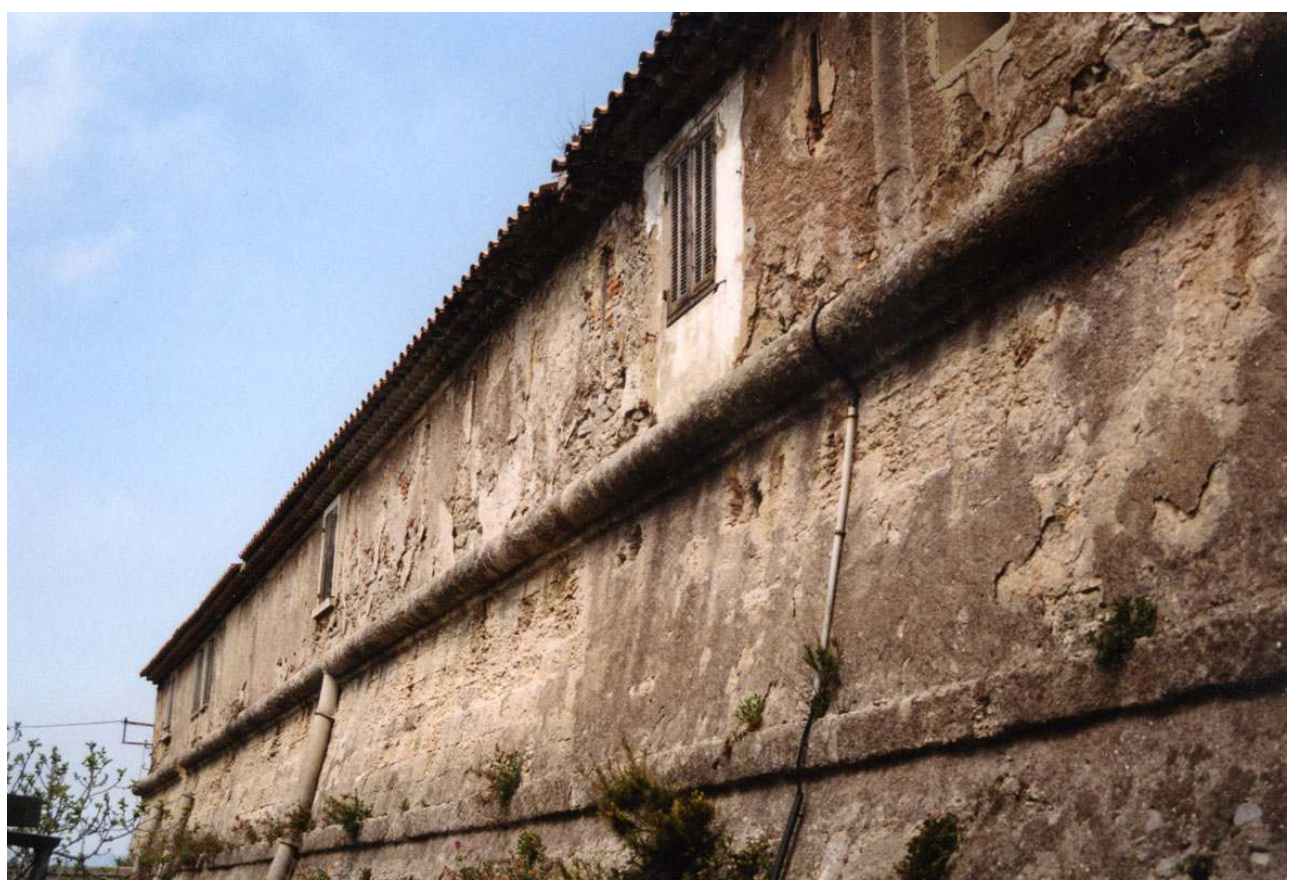

Aile gauche du fort de l'Eguillette, avant travaux. Phot. B. Cros. 


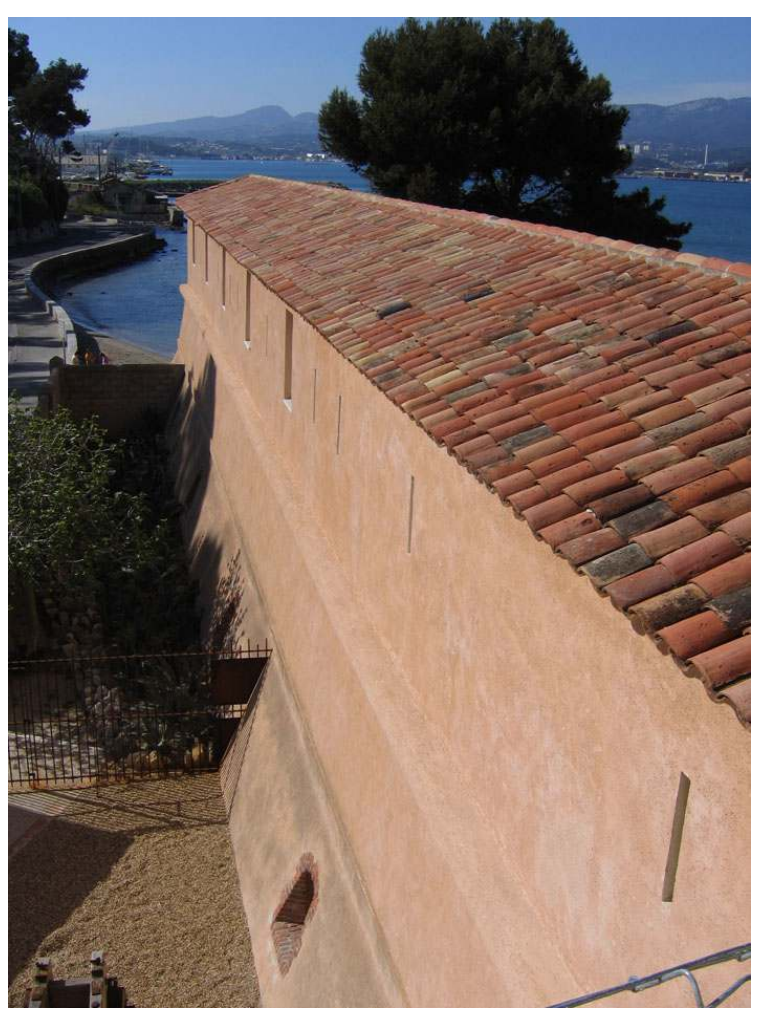

Aile gauche du fort de l'Eguillette, après travaux. Phot. B. Cros.

\section{Une démarche novatrice en matière de protection et d'action}

Sur le plan réglementaire, les démarches ont connu une évolution significative au début des années 2000. La ville de Toulon était alors dotée d'une zone de protection du patrimoine architectural, urbain et paysager (ZPPAUP) couvrant la vieille ville, dont l'urbanisation actuelle est le fruit d'une période allant du Moyen Âge au début du XIX ${ }^{\mathrm{e}}$ siècle pour l'essentiel. Des témoins significatifs de l'époque la plus ancienne au XVIII siècle jalonnent particulièrement ce tissu urbain. Le périmètre de la ZPPAUP empiétait largement sur l'arsenal, sans raison explicite et sans réelle portée pratique. Par ailleurs, l'arsenal de la marine est, en vertu du Code de l'urbanisme, dispensé de toute procédure en matière d'autorisation de construire. De concert avec l'architecte des Bâtiments de France $(\mathrm{ABF})$, une démarche était entamée afin de restreindre d'une part la zone périphérique de la ZPPAUP empiétant sur l'arsenal et, d'autre part, de créer au sein de l'arsenal des ZPPAUP couvrant les différents ensembles patrimoniaux reconnus dignes d'intérêt. Particulièrement novatrice au sein de la Défense, cette démarche concernait l'ensemble des édifices ou ouvrages portuaires récapitulés dans le tableau qui précède et s'étendait à plusieurs constructions des $\mathrm{XIX}^{\mathrm{e}}$ et $\mathrm{XX}^{\mathrm{e}}$ siècles. Les ZPPAUP ainsi créées sont soit strictement superposées à un édifice et ses abords immédiats, soit élargies à un périmètre assurant une cohérence " urbaine » à la place prise par l'ouvrage protégé. Dans ce dernier type de situation, on peut signaler les remparts de Malbousquet, qui sont considérés dans leur continuité et leur profondeur, ou bien l'ancien Dépôt des équipages, 
pris dans sa globalité, espaces découverts inclus. En prenant le parti de soumettre à l'avis conforme de l'ABF les interventions visant des constructions non protégées au titre des Monuments historiques et affranchies des procédures du Code de l'urbanisme ${ }^{17}$, la marine nationale s'engage en fin de compte à respecter devant une autorité « tierce » la « dignité d'intérêt » conférée de manière collégiale à des éléments significatifs et relativement nombreux du patrimoine de la base navale. Entrées en vigueur au printemps 2007, au moment de la validation de la , ces dispositions ne sont pas demeurées au stade des intentions de principe. Dès l'été 2007, plusieurs projets de constructions, impactant différentes ZPPAUP ont fait l'objet de saisine de l'ABF. Des mises au point demandées par celui-ci ont été intégrées à plusieurs projets. Dans certains cas, c'est dès le stade des études de faisabilité qu'ont été étudiées des options de mise en valeur qui, sans l'existence des ZPPAUP, n'auraient probablement pas été examinées et auraient même vraisemblablement conduit à des démolitions.

\section{BIBLIOGRAPHIE}

ACADÉMIE DU VAR. Toulon ville forte, sept siècles de fortifications. Toulon : Autres temps, 2007.

ACADÉMIE DU VAR. Toulon et son patrimoine, Portes et façades, Corderie et front de mer. Toulon : Autres temps, 2008.

CROS, Bernard. Citadelles d'azur, quatre siècles d'architecture militaire varoise. Aix-en-

Provence : ÉDISUD, 1998.

Dossier « Patrimoine maritime », Monumental. Paris : Monum, Éditions du patrimoine, 2003.

MARINE NATIONALE. Pierres de mer, le patrimoine immobilier de la marine nationale. Paris : ADDIM, 1996.

TRUTTMANN, Philippe. Canons et murailles, catalogue d'exposition. La Seyne-sur-Mer, 1989.

HENRY. Mémoire sur l'état primitif de la ville de Toulon et de son port, et sur leurs agrandissements successifs. Bulletin de l'Académie du Var, (AV), Toulon, 1850.

HENRY. Fortification de Toulon sous Henry IV. Bulletin de l'Académie du Var, (AV), 1853 et 1855.

LAMBERT, Gustave. Agrandissements et fortifications de la ville de Toulon. Bulletin de l'Académie du Var, (AV), 1873.

PETIT, Raymond. Survol des fortifications de Toulon. Bulletin de la société des Amis du vieux Toulon, Toulon, 1988. 


\section{NOTES}

1. - La ville est protégée par son enceinte bastionnée de 1595, qui se prolonge en mer par les môles fortifiés créés par Bonnefons. C'est dans l'angle inférieur droit de la darse que l'arsenal de marine sera fondé.

2. - L'étendue et la protection naturelle de la rade lui confèrent un caractère exceptionnel sur la côte provençale.

3. - Conçus par l'ingénieur des Travaux hydrauliques de la marine Honoré Bernard, les pavillons symétriques de l'entrée témoignent d'une instruction marquée par les réflexions de Ledoux. Construite selon un style Néo-antique, la chapelle est implantée en un point qui forme belvédère et point remarquable au sein des jardins qui dominent la rade.

4. - Les agrandissements successifs de l'arsenal vers l'ouest sont mis en évidence par la création d'une nouvelle darse à chaque étape.

5. - L'emprise actuelle de la marine sur la rade correspond à l'ensemble du littoral toulonnais Seule une «fenêtre » de $600 \mathrm{~m}$ marque le rivage non militaire, au niveau du port de commerce. Le plan d'eau, dans sa quasi-totalité, dépend de l'autorité maritime militaire.

6. - Défavorisée par un environnement immédiat de type routier, partiellement détruite par un incendie en 1907 et bombardée durant la guerre, la corderie constitue un élément marquant du patrimoine maritime toulonnais, tant par ses dimensions que par son histoire.

7. - En service depuis plus de deux siècles, le bassin n'a pratiquement pas été modifié depuis sa construction. Ses structures sont en parfait état de conservation.

8. - Le bâtiment est pratiquement dans son état originel de la fin du XVII ${ }^{\mathrm{e}}$ siècle. Il bénéficie désormais d'une Zone de protection de type ZPPAUP.

9. - Initialement dénommée Commission permanente du patrimoine naval et aéronaval (CPPNA), elle devient Commission du patrimoine de la marine lorsque l'acronyme CPM utilisé par une autre entité devient disponible.

10. - Conçu par Vauban, le fort a été sévèrement endommagé durant le siège de 1707. Reconstruit pratiquement à l'identique en 1708 , il est aujourd'hui inscrit à l'inventaire supplémentaire des Monuments historiques.

11. - De nombreuses tours sont construites dans les années 1635, du Brusc à Antibes en passant par les îles d'Hyères.

12. - Dix batteries aménagées selon les normes de 1846 sont encore visibles, plus ou moins remaniées depuis.

13. - La campagne de restauration conduite par la marine avec les Monuments historiques à partir de 1994 a permis de recréer les ventilations naturelles propres à assainir le monument.

14. - Association TREMPLIN (TRavail EMPLoi INsertion).

15. - Adjonction d'un logement de gardien contre la tour, aménagements propres au fonctionnement d'un parc à combustibles contigu et dont le fort servait d'annexe.

16. - L'expérience conduite depuis 1996 au fort de l'Eguillette est exemplaire à plus d'un titre. Sa réussite profite simultanément à la Défense, maître d'ouvrage, aux personnes en chantier d'insertion et au public qui peut accéder à ce lieu à vocation culturelle.

17. - Les démolitions sont cependant soumises à la procédure de permis au titre du code de l'urbanisme. 


\section{RÉSUMÉS}

La marine nationale est présente à Toulon depuis quatre siècles. Au fil du temps, elle a édifié autour de la rade un patrimoine portuaire et fortifié en rapport avec l'importance stratégique de son implantation méditerranéenne. Actuellement, une grande partie de ce patrimoine est toujours utilisée par la Marine. Une très faible partie est protégée au titre des Monuments historiques. L'article décrit les méthodes mises en œuvre pour identifier et préserver ce patrimoine.

The French navy has been present at Toulon for four centuries. Over this period, a considerable heritage of port installations and fortifications has developed around the roadstead, in keeping with the strategic importance of Toulon on the Mediterranean. Today, much of this heritage is still in use for the requirements of the navy, and very little of it enjoys statutory historic monuments protection. The article describes the methods applied in the evaluation of this heritage and the policies implemented for its preservation.

\section{INDEX}

Mots-clés : arsenaux maritimes, fortifications côtières, patrimoine maritime, patrimoine militaire, politique patrimoniale

Keywords : coastal defences, heritage policy, military heritage, naval dockyards, naval heritage

\section{AUTEUR}

\section{BERNARD CROS}

ingénieur en chef de la marine (e.r.) Ancien officier Patrimoine du service des Travaux maritimes, Ancien chef de département à la direction du Service d'infrastructure de la défense à Toulon e.cros.sanary@wanadoo.fr 